\title{
Hydrovinylation of olefins catalyzed by an Iridium Complex via C-H
}

\author{
Activation \\ Gaurav Bhalla, ${ }^{\ddagger}$ Jonas Oxgaard, ${ }^{\dagger}$ William A. Goddard, $I I I,{ }^{\dagger}$ and Roy A. Periana ${ }^{* \neq}$ \\ ${ }^{*}$ Loker Hydrocarbon Research Institute, Department of Chemistry, University of \\ Southern California, CA 90089. \\ ${ }^{\dagger}$ Materials and Process Simulation Center, Beckman Institute, Division of Chemistry and Chemical \\ Engineering, California Institute of Technology, CA 91125.
}

General Considerations: All air and water sensitive procedures were carried out either in an inert atmosphere glove box or using standard Schlenk techniques. All solvents used were reagent grade. THF was dried over sodium/benzophenone ketyl and distilled under nitrogen. All deuterated solvents (Cambridge Isotopes), $\mathrm{Hg}(\operatorname{vinyl})_{2}$ (Organometallics, Inc.) and $\mathrm{IrCl}_{3} \cdot \mathrm{xH}_{2} \mathrm{O}$ (Pressure chemicals) were used as received. GC/MS analysis was performed on a Shimadzu GC-MS QP5000 (ver. 2) equipped with cross-linked methyl silicone gum capillary column (DB5). The retention times of the products were confirmed by comparison to authentic samples. NMR spectra were obtained on a Bruker AC-250 spectrometer $\left\{250.13 \mathrm{MHz}\left({ }^{1} \mathrm{H}\right)\right.$ and $\left.62.90 \mathrm{MHz}\left({ }^{13} \mathrm{C}\right)\right\}$, Bruker AM-360 spectrometer $\left\{360.14 \mathrm{MHz}\left({ }^{1} \mathrm{H}\right)\right.$ and $\left.90.57 \mathrm{MHz}\left({ }^{13} \mathrm{C}\right)\right\}$ or on a Varian Mercury $400\left\{400.151 \mathrm{MHz}\left({ }^{1} \mathrm{H}\right)\right.$ and $\left.100.631 \mathrm{MHz}\left({ }^{13} \mathrm{C}\right)\right\}$ spectrometer. All coupling constants are reported in units of $\mathrm{Hz}$. Elemental analyses were done by Desert Analytics Laboratory; Arizona.

X-ray Crystallography. X-ray Crystallography. Diffraction data for Vinyl-IrPy was collected at low temperature $(T=143 \mathrm{~K})$ on a Bruker SMART APEX CCD diffractometer with graphite-monochromated Mo K $\alpha$ radiation $(\lambda=0.71073 \AA$ ). The cell parameters for the Ir complex were obtained from the least-squares refinement of the spots (from 60 collected frames) using the SMART program. A hemisphere of the crystal data was collected up to a resolution of $0.75 \AA$, and the intensity data was processed using the Saint Plus program. All calculations for structure determination were carried out using the SHELXTL package (version 5.1). ${ }^{1}$ Initial atomic positions were located by direct methods using XS, and the structure was refined by least-squares methods using

${ }^{1}$ Sheldrick, G. M. SHELXTL, version5.1; Bruker Analytical X-ray System, Inc.: Madison, WI, 1997. 
SHELX with 3503 independent reflections and within the range of $\Phi 2.71-26.36^{\circ}$ (completeness $100 \%$ ). Absorption corrections were applied by using SADABS. ${ }^{2}$ Calculated hydrogen positions were input and refined in a riding manner along with the attached carbons.

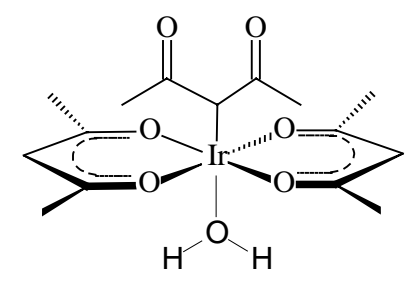

\section{Synthesis of $\left.\left[\mathrm{C}^{3} \text {-acac-Ir(O,O-acac }\right)_{2}\left(\mathrm{H}_{2} \mathrm{O}\right)\right]\left(\operatorname{Acac}-\mathrm{Ir}-\mathrm{H}_{2} \mathrm{O}\right)$ :}

In a $100 \mathrm{~mL}$ round-bottom flask equipped with a reflux condenser vented to an oil bubbler, $1 \mathrm{~g}$ of $\operatorname{IrCl}_{3}\left(\mathrm{H}_{2} \mathrm{O}\right)_{\mathrm{x}}(54.11 \%$ of $\mathrm{Ir}, 2.82 \mathrm{mmol}), 10 \mathrm{~mL}$ of 2,4-pentanedione $(9.75$ mmol) and $1 \mathrm{~g}$ of $\mathrm{NaHCO}_{3}(11.9 \mathrm{mmol})$ were added. The mixture was heated to gentle reflux with stirring for $40 \mathrm{~h}$. During this time, a yellow solid precipitated. The reaction mixture was cooled to room temperature and the solid was collected as crude product. The precipitate was thoroughly washed with dichloromethane to remove excess 2,4pentanedione and $\operatorname{Ir}(\mathrm{acac})_{3}$. The washed yellow solid was dissolved in $200 \mathrm{~mL} \mathrm{H}_{2} \mathrm{O}$ at room temperature under vigorous stirring and was gravity filtered to remove any undissolved material. Solution was concentrated by blowing air through the solution to give Acac-Ir-H $\mathbf{H}_{2} \mathbf{O}$ as yellow solid (500 $\mathrm{mg}, 35 \%$ yield) complex. ${ }^{1} \mathrm{H}-\mathrm{NMR}$ $\left(\mathrm{D}_{2} \mathrm{O} / 10 \% \mathrm{CD}_{3} \mathrm{OD}\right): \delta 5.53\left(\mathrm{~s}, 2 \mathrm{H}, \mathrm{O}-\mathrm{acac}^{3} \mathrm{C}^{3} H\right), 5.47\left(\mathrm{~s}, 1 \mathrm{H}, \mathrm{C}^{3}\right.$-acac-C $\left.\mathrm{C}^{3} H\right), 1.90(\mathrm{~s}, 12 \mathrm{H}$, $\mathrm{O}$-acac- $\left.\mathrm{CH}_{3}\right), 1.73\left(\mathrm{~s}, 6 \mathrm{H}, \mathrm{C}^{3}\right.$-acac-CH$) .{ }^{13} \mathrm{C}\left\{{ }^{1} \mathrm{H}\right\}$ NMR $\left(\mathrm{D}_{2} \mathrm{O} / 10 \% \mathrm{CD}_{3} \mathrm{OD}\right): \delta 217.9\left(\mathrm{C}^{3}-\right.$ acac $C=\mathrm{O}), 188.0(\mathrm{O}$-acac $C=\mathrm{O}), 104.5\left(\mathrm{O}\right.$-acac- $\left.C^{3} \mathrm{H}\right), 50.0\left(\mathrm{C}^{3}\right.$-acac- $\left.C^{3} \mathrm{H}\right), 33.2\left(\mathrm{C}^{3}\right.$-acac $\left.\mathrm{CH}_{3}\right)$, 28.3(O-acac $\left.\mathrm{CH}_{3}\right)$. Anal. Calcd. for $\mathrm{C}_{15} \mathrm{H}_{23} \mathrm{O}_{7} \mathrm{Ir}$ : C, 35.50; H, 4.57. Found: C, $35.28 ; \mathrm{H}, 4.60$.

\footnotetext{
${ }^{2}$ Blessing, R. H. Acta Crystallogr. 1995, A51, 33-38.
} 


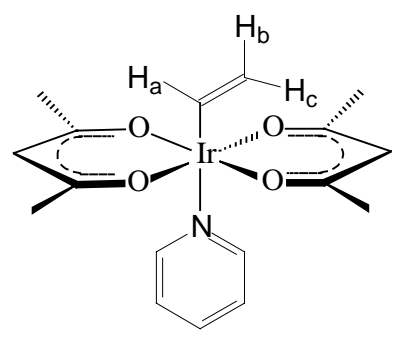

2. Synthesis of $\left[\mathrm{CH}_{2}=\mathrm{CH}-\operatorname{Ir}(\mathrm{O}, \mathrm{O}-\mathrm{acac})_{2}(\mathrm{Py})\right]$ (Vinyl-Ir-Py) :

To a mixture containing $100 \mathrm{mg}(0.197 \mathrm{mmol})$ of Acac-Ir- $\mathbf{H}_{2} \mathbf{O}$ in $10 \mathrm{~mL}$ of methanol, $100 \mu \mathrm{L}(\sim 0.40 \mathrm{mmol})$ of divinylmercury, $\left[\mathrm{Hg}\left(\mathrm{CH}=\mathrm{CH}_{2}\right)_{2}\right]$ was added. $\left\{\left[\mathrm{Hg}\left(\mathrm{CH}=\mathrm{CH}_{2}\right)_{2}\right]\right.$ is a stench and a highly toxic material, handle with care! When handling this chemical impervious gloves and a face shield a minimum of 8 inches in length must be worn. Also work should be carried out in a well ventilated hood. Importantly, latex, neoprene and butyl gloves DO NOT provide suitable protection against this material. Permeability tests have shown that Silver Shield laminate gloves are impermeable to dimethylmercury for at least 4 hours. The Silver Shield glove should be worn under an outer glove that would be resistant to abrasion and tears. The vial containing the divinylmercury should be clamped and the contents removed by means of a glass syringe and Schlenk Techniques. Gloves should be removed and disposed in a manner that precludes re-entry of this material into the workplace and in accordance with the requirements of the State Hazardous Waste Regulatory Authority.\} The mixture was stirred at room temperature for $2 \mathrm{~h}$ under Argon. During this the formation of elemental mercury was observed and the color of the solution changed from orange to yellow. To this $100 \mu \mathrm{L}(1.26 \mathrm{mmol})$ of pyridine was added and stirred for 10 minutes. The solution was vacuum transferred and the distillate was disposed off after treatment with conc. nitric acid. The resulting yellow precipitate was then redissolved in methanol and cooled slowly with dry ice/acetone mixture, whereby yellow needles were formed. The solution was decanted and the solid was thoroughly washed with hexanes, followed by ether. The x-ray quality crystals were formed by the diffusion of hexanes into a concentrated solution of $\mathrm{CH}_{2} \mathrm{Cl}_{2}$ and obtained in $40 \%\left(40 \mathrm{mg}\right.$ ) yield. ${ }^{1} \mathrm{H}$ NMR $\left(\mathrm{CDCl}_{3}\right): \delta 8.42\left(\mathrm{~d},{ }^{3} \mathrm{~J}=5.3,2 \mathrm{H}, o-H \mathrm{py}\right), 8.20\left(\mathrm{dd},{ }^{3} \mathrm{~J}_{\mathrm{ab}}=\right.$ $\left.9.7,{ }^{3} \mathrm{~J}_{\mathrm{ac}}=17.7,1 \mathrm{H}, \mathrm{C} H_{a}-\mathrm{Ir}\right), 7.80\left(\mathrm{tt},{ }^{3} \mathrm{~J}=8.0,{ }^{4} \mathrm{~J}=1.8,1 \mathrm{H}, \mathrm{p}-H\right.$ py), $7.36(\mathrm{~m}, 2 \mathrm{H}, m-H$ py), $5.28\left(\mathrm{~s}, 2 \mathrm{H}\right.$, acac-CH), 5.22(dd, $\left.{ }^{3} \mathrm{~J}_{\mathrm{ab}}=9.7,{ }^{3} \mathrm{~J}_{\mathrm{bc}}=2.7,1 \mathrm{H}, \mathrm{H}_{\mathrm{b}}\right), 4.74\left(\mathrm{dd},{ }^{3} \mathrm{~J}_{\mathrm{ac}}=17.7,{ }^{3} \mathrm{~J}_{\mathrm{bc}}\right.$ $\left.=2.7,1 \mathrm{H}, \mathrm{H}_{\mathrm{c}}\right), 1.83\left(\mathrm{~s}, 12 \mathrm{H}\right.$, acac- $\left.\mathrm{CH}_{3}\right) \cdot{ }^{13} \mathrm{C}\left\{{ }^{1} \mathrm{H}\right\}\left(\mathrm{CDCl}_{3}\right): \delta 184.56($ acac $C=\mathrm{O})$, 149.95(o-Py), 137.51 (p-Py), 127.13( $\left(\mathrm{Ir}-\mathrm{CH}=\mathrm{CH}_{2}\right.$ ), 125.10 (m-Py), $118.63\left(\mathrm{Ir}-\mathrm{CH}=\mathrm{CH}_{2}\right.$ ), 
102.92 (acac- $\left.C^{3} \mathrm{H}\right), 27.22$ (acac- $\mathrm{CH}_{3}$ ). Anal. Calcd for $\mathrm{C}_{17} \mathrm{H}_{22} \mathrm{NO}_{4} \mathrm{Ir}$ : C, 41.12; $\mathrm{H}, 4.47$; N, 2.82. Found: C, 41.38; H, 4.95; N, 2.70.

Catalytic hydrovinylation with Ethylene using $\mathbf{C H}_{3}$-Ir-Py or Vinyl-Ir-Py: A $3 \mathrm{~mL}$ stainless steel autoclave, equipped with a glass insert and a magnetic stir bar was charged with $1 \mathrm{~mL}$ of distilled hexaflourobenzene and $10 \mathrm{mg}(5 \mathrm{mM}, \sim 0.1 \mathrm{~mol} \%)$ of catalyst $\left(\mathbf{C H}_{3}\right.$-Ir-Py or Vinyl-Ir-Py). The reactor was degassed with argon, pressurized with 2.96 $\mathrm{MPa}$ of ethylene. The autoclave was heated for $1 \mathrm{~h}$ in a well-stirred heating bath maintained at $180{ }^{\circ} \mathrm{C}$. The liquid phase was sampled and the product yields were determined by GC-MS using methyl cyclohexane as an internal standard, introduced into the reaction solution after the reaction.

Catalytic hydrovinylation with Propylene using $\mathbf{C H}_{3}$-Ir-Py or Vinyl-Ir-Py: A $3 \mathrm{~mL}$ stainless steel autoclave, equipped with a glass insert and a magnetic stir bar was charged with $1 \mathrm{~mL}$ of distilled hexaflourobenzene and $10 \mathrm{mg}(5 \mathrm{mM}, \sim 0.1 \mathrm{~mol} \%)$ of catalyst ( $\mathbf{C H}_{3}$-Ir-Py or Vinyl-Ir-Py). The reactor was degassed with argon, pressurized with 0.96 MPa of propylene with an additional $2.96 \mathrm{MPa}$ of argon. The autoclave was heated for 3 $\mathrm{h}$ in a well stirred heating bath maintained at $180^{\circ} \mathrm{C}$. The liquid phase was sampled and the product yields were determined by GC-MS using methyl cyclohexane as an internal standard, introduced into the reaction solution after the reaction.

Stoichiometric reaction of Ethylene with $\mathrm{CH}_{3}$-Ir-Py: A solution of $\mathrm{CH}_{3}$-Ir-Py in $\mathrm{C}_{6} \mathrm{D}_{12}(5 \mathrm{mM})$ was made with trimethoxybenzene (internal standard) and transferred to an oven-dried high pressure NMR tube fitted with a valve. Ethylene (4 MPa) was added to this NMR tube at room temperature and subjected to NMR studies. The formation of the vinyl-Ir-Py (verified by independent synthetic route) was standardized at different temperatures and was finally heated for $15 \mathrm{~h}$ at $150{ }^{\circ} \mathrm{C}$. 
Table 1. Crystal data and structure refinement for $\mathrm{C}_{17} \mathrm{H}_{22}$ Ir $\mathrm{N} \mathrm{O}_{4}$. (Vinyl-Ir-Py) Identification code irvinylm

Empirical formula

Formula weight

Temperature

Wavelength

Crystal system

Space group

Unit cell dimensions

Volume

Z

Density (calculated)

Absorption coefficient

$\mathrm{F}(000)$

Crystal size

Theta range for data collection

Index ranges

Reflections collected

Independent reflections

Completeness to theta $=26.36^{\circ}$

Transmission factors

Refinement method

Data / restraints / parameters
C17 H22 Ir N O4

496.56

143(2) K

$0.71073 \AA$

Trigonal

P3(2)

$$
\begin{array}{ll}
\mathrm{a}=8.2247(8) \AA & \alpha=90^{\circ} . \\
\mathrm{b}=8.2247(8) \AA & \beta=90^{\circ} . \\
\mathrm{c}=22.586(4) \AA & \gamma=120^{\circ} .
\end{array}
$$

1323.2(3) $\AA^{3}$

3

$1.869 \mathrm{Mg} / \mathrm{m}^{3}$

$7.586 \mathrm{~mm}^{-1}$

720

$0.23 \times 0.16 \times 0.02 \mathrm{~mm}^{3}$

2.71 to $26.36^{\circ}$.

$-7<=\mathrm{h}<=10,-10<=\mathrm{k}<=8,-28<=\mathrm{l}<=26$

7624

$3503[\mathrm{R}(\mathrm{int})=0.0571]$

$100.0 \%$

$\mathrm{min} /$ max ratio: 0.521

Full-matrix least-squares on $\mathrm{F}^{2}$

3503 / 20 / 103 
Goodness-of-fit on $\mathrm{F}^{2}$

Final $\mathrm{R}$ indices [I $>2 \operatorname{sigma}(\mathrm{I})]$

$\mathrm{R}$ indices (all data)

Largest diff. peak and hole
1.146

$\mathrm{R} 1=0.0668, \mathrm{wR} 2=0.1611$

$\mathrm{R} 1=0.0713, \mathrm{wR} 2=0.1629$

2.610 and -6.542 e. $\AA^{-3}$ 
Table 2. Atomic coordinates ( $\times 10^{4}$ ) and equivalent isotropic displacement parameters $\left(\AA^{2} \mathrm{x} 10^{3}\right)$

for $\mathrm{C} 17 \mathrm{H} 22 \mathrm{Ir} \mathrm{N} \mathrm{O} 4 . \mathrm{U}(\mathrm{eq})$ is defined as one third of the trace of the orthogonalized $\mathrm{U}^{\mathrm{ij}}$ tensor.

\begin{tabular}{|c|c|c|c|c|}
\hline & $\mathrm{X}$ & $\mathrm{y}$ & $\mathrm{Z}$ & $\mathrm{U}(\mathrm{eq})$ \\
\hline $\operatorname{Ir}(1)$ & $6440(1)$ & $10206(1)$ & $5006(3)$ & $29(1)$ \\
\hline $\mathrm{O}(1)$ & $4510(30)$ & $10070(20)$ & $5570(7)$ & $30(4)$ \\
\hline $\mathrm{O}(2)$ & $6430(40)$ & $12220(30)$ & $4468(10)$ & $51(7)$ \\
\hline $\mathrm{O}(3)$ & $6460(20)$ & $8230(20)$ & $5510(7)$ & $26(4)$ \\
\hline $\mathrm{O}(4)$ & $8350(30)$ & $10330(30)$ & $4407(9)$ & $49(6)$ \\
\hline $\mathrm{N}(1)$ & $4230(20)$ & $8080(20)$ & $4429(5)$ & $33(6)$ \\
\hline $\mathrm{C}(1)$ & $2550(40)$ & $11050(40)$ & $6005(12)$ & $56(7)$ \\
\hline$C(2)$ & $3860(30)$ & $11200(30)$ & $5525(10)$ & $49(6)$ \\
\hline $\mathrm{C}(3)$ & $3980(30)$ & $12390(30)$ & $5046(8)$ & $41(5)$ \\
\hline$C(4)$ & $5340(30)$ & $12870(30)$ & $4583(10)$ & $42(5)$ \\
\hline$C(5)$ & $5490(40)$ & $14260(30)$ & $4124(11)$ & $51(6)$ \\
\hline$C(6)$ & $7620(40)$ & $6340(30)$ & 5894(11) & $51(6)$ \\
\hline $\mathrm{C}(7)$ & $7630(30)$ & $7700(30)$ & $5442(9)$ & $32(5)$ \\
\hline $\mathrm{C}(8)$ & $8940(30)$ & $8270(30)$ & 4988(8) & $48(6)$ \\
\hline $\mathrm{C}(9)$ & $9200(30)$ & $9430(30)$ & $4512(9)$ & $35(5)$ \\
\hline$C(10)$ & $10560(30)$ & $9710(40)$ & 4018(11) & $52(6)$ \\
\hline $\mathrm{C}(11)$ & $2620(20)$ & $6640(30)$ & $4654(5)$ & $45(5)$ \\
\hline $\mathrm{C}(12)$ & $1270(30)$ & $5390(30)$ & $4285(6)$ & $52(6)$ \\
\hline$C(13)$ & $1500(30)$ & $5620(30)$ & $3691(6)$ & $57(6)$ \\
\hline$C(14)$ & $3100(20)$ & $7070(30)$ & $3463(5)$ & $50(6)$ \\
\hline$C(15)$ & $4450(20)$ & $8310(20)$ & $3834(5)$ & $40(5)$ \\
\hline$C(16)$ & $8400(40)$ & $12110(40)$ & $5521(11)$ & $34(7)$ \\
\hline $\mathrm{C}(17)$ & $8640(40)$ & $12360(40)$ & $6093(13)$ & $63(7)$ \\
\hline
\end{tabular}


Table 3. Bond lengths $[\AA]$ and angles $\left[{ }^{\circ}\right]$ for $\mathrm{C} 17 \mathrm{H} 22$ Ir N O4.

\begin{tabular}{lc}
\hline $\operatorname{Ir}(1)-\mathrm{C}(16)$ & $1.97(3)$ \\
$\mathrm{Ir}(1)-\mathrm{O}(3)$ & $1.988(16)$ \\
$\mathrm{Ir}(1)-\mathrm{O}(1)$ & $1.994(18)$ \\
$\mathrm{Ir}(1)-\mathrm{O}(4)$ & $2.04(2)$ \\
$\mathrm{Ir}(1)-\mathrm{O}(2)$ & $2.06(2)$ \\
$\mathrm{Ir}(1)-\mathrm{N}(1)$ & $2.209(14)$ \\
$\mathrm{O}(1)-\mathrm{C}(2)$ & $1.29(3)$ \\
$\mathrm{O}(2)-\mathrm{C}(4)$ & $1.28(3)$ \\
$\mathrm{O}(3)-\mathrm{C}(7)$ & $1.26(2)$ \\
$\mathrm{O}(4)-\mathrm{C}(9)$ & $1.27(2)$ \\
$\mathrm{N}(1)-\mathrm{C}(15)$ & $1.356(13)$ \\
$\mathrm{N}(1)-\mathrm{C}(11)$ & $1.358(13)$ \\
$\mathrm{C}(1)-\mathrm{C}(2)$ & $1.49(2)$ \\
$\mathrm{C}(2)-\mathrm{C}(3)$ & $1.43(2)$ \\
$\mathrm{C}(3)-\mathrm{C}(4)$ & $1.43(2)$ \\
$\mathrm{C}(4)-\mathrm{C}(5)$ & $1.50(2)$ \\
$\mathrm{C}(6)-\mathrm{C}(7)$ & $1.51(2)$ \\
$\mathrm{C}(7)-\mathrm{C}(8)$ & $1.39(2)$ \\
$\mathrm{C}(8)-\mathrm{C}(9)$ & $1.38(2)$ \\
$\mathrm{C}(9)-\mathrm{C}(10)$ & $1.51(2)$ \\
$\mathrm{C}(11)-\mathrm{C}(12)$ & $1.357(13)$ \\
$\mathrm{C}(12)-\mathrm{C}(13)$ & $1.355(13)$ \\
$\mathrm{C}(13)-\mathrm{C}(14)$ & $1.360(13)$ \\
$\mathrm{C}(14)-\mathrm{C}(15)$ & $1.358(13)$ \\
$\mathrm{C}(16)-\mathrm{C}(17)$ & $1.31(4)$ \\
$\mathrm{C}(16)-\operatorname{Ir}(1)-\mathrm{O}(3)$ & $88.4(9)$ \\
$\mathrm{C}(16)-\operatorname{Ir}(1)-\mathrm{O}(1)$ & $88.9(9)$ \\
$\mathrm{O}(3)-\operatorname{Ir}(1)-\mathrm{O}(1)$ & $85.3(6)$ \\
$\mathrm{C}(16)-\mathrm{Ir}(1)-\mathrm{O}(4)$ & $92.7(11)$ \\
$\mathrm{O}(3)-\operatorname{Ir}(1)-\mathrm{O}(4)$ & $95.8(8)$ \\
$\mathrm{O}(1)-\mathrm{Ir}(1)-\mathrm{O}(4)$ & $178.1(9)$ \\
$\mathrm{C}(16)-\mathrm{Ir}(1)-\mathrm{O}(2)$ & $92.3(12)$ \\
$\mathrm{O}(3)-\mathrm{Ir}(1)-\mathrm{O}(2)$ & $178.8(9)$ \\
&
\end{tabular}




$\begin{array}{lc}\mathrm{O}(1)-\mathrm{Ir}(1)-\mathrm{O}(2) & 95.7(8) \\ \mathrm{O}(4)-\mathrm{Ir}(1)-\mathrm{O}(2) & 83.2(8) \\ \mathrm{C}(16)-\mathrm{Ir}(1)-\mathrm{N}(1) & 179.6(13) \\ \mathrm{O}(3)-\mathrm{Ir}(1)-\mathrm{N}(1) & 91.8(8) \\ \mathrm{O}(1)-\mathrm{Ir}(1)-\mathrm{N}(1) & 90.9(7) \\ \mathrm{O}(4)-\mathrm{Ir}(1)-\mathrm{N}(1) & 87.5(7) \\ \mathrm{O}(2)-\mathrm{Ir}(1)-\mathrm{N}(1) & 87.4(8) \\ \mathrm{C}(2)-\mathrm{O}(1)-\mathrm{Ir}(1) & 121.0(14) \\ \mathrm{C}(4)-\mathrm{O}(2)-\mathrm{Ir}(1) & 120.1(18) \\ \mathrm{C}(7)-\mathrm{O}(3)-\mathrm{Ir}(1) & 122.4(14) \\ \mathrm{C}(9)-\mathrm{O}(4)-\mathrm{Ir}(1) & 118.9(16) \\ \mathrm{C}(15)-\mathrm{N}(1)-\mathrm{C}(11) & 119.8(6) \\ \mathrm{C}(15)-\mathrm{N}(1)-\mathrm{Ir}(1) & 118.3(8) \\ \mathrm{C}(11)-\mathrm{N}(1)-\mathrm{Ir}(1) & 121.7(8) \\ \mathrm{O}(1)-\mathrm{C}(2)-\mathrm{C}(3) & 130(2) \\ \mathrm{O}(1)-\mathrm{C}(2)-\mathrm{C}(1) & 115(2) \\ \mathrm{C}(3)-\mathrm{C}(2)-\mathrm{C}(1) & 114(2) \\ \mathrm{C}(2)-\mathrm{C}(3)-\mathrm{C}(4) & 122(2) \\ \mathrm{O}(2)-\mathrm{C}(4)-\mathrm{C}(3) & 130(2) \\ \mathrm{O}(2)-\mathrm{C}(4)-\mathrm{C}(5) & 112(2) \\ \mathrm{C}(3)-\mathrm{C}(4)-\mathrm{C}(5) & 118(2) \\ \mathrm{O}(3)-\mathrm{C}(7)-\mathrm{C}(8) & 125.1(18) \\ \mathrm{O}(3)-\mathrm{C}(7)-\mathrm{C}(6) & 117.1(19) \\ \mathrm{C}(8)-\mathrm{C}(7)-\mathrm{C}(6) & 117.8(19) \\ \mathrm{C}(9)-\mathrm{C}(8)-\mathrm{C}(7) & 129(2) \\ \mathrm{O}(4)-\mathrm{C}(9)-\mathrm{C}(8) & 127(2) \\ \mathrm{O}(4)-\mathrm{C}(9)-\mathrm{C}(10) & 111(2) \\ \mathrm{C}(8)-\mathrm{C}(9)-\mathrm{C}(10) & 122(2) \\ \mathrm{C}(12)-\mathrm{C}(11)-\mathrm{N}(1) & 120.1(6) \\ \mathrm{C}(13)-\mathrm{C}(12)-\mathrm{C}(11) & 120.0(6) \\ \mathrm{C}(12)-\mathrm{C}(13)-\mathrm{C}(14) & 120.1(5) \\ \mathrm{C}(15)-\mathrm{C}(14)-\mathrm{C}(13) & 119.7(6) \\ \mathrm{N}(1)-\mathrm{C}(15)-\mathrm{C}(14) & 120.2(6) \\ \mathrm{C}(17)-\mathrm{C}(16)-\mathrm{Ir}(1) & 135(2) \\ & \\ & \end{array}$

Symmetry transformations used to generate equivalent atoms: 
Table 4. Anisotropic displacement parameters $\left(\AA^{2} \times 10^{3}\right)$ for $\mathrm{C} 17 \mathrm{H} 22 \mathrm{Ir} \mathrm{N} \mathrm{O} 4$. The anisotropic

displacement factor exponent takes the form: $-2 \pi^{2}\left[\mathrm{~h}^{2} \mathrm{a}^{* 2} \mathrm{U}^{11}+\ldots+2 \mathrm{~h} \mathrm{k} \mathrm{a} \mathrm{a}^{*} \mathrm{U}^{12}\right]$

\begin{tabular}{|c|c|c|c|c|c|c|}
\hline & $\mathrm{U}^{11}$ & $\mathrm{U}^{22}$ & $\mathrm{U}^{33}$ & $\mathrm{U}^{23}$ & $\mathrm{U}^{13}$ & $\mathrm{U}^{12}$ \\
\hline $\operatorname{Ir}(1)$ & $26(1)$ & $31(1)$ & $33(1)$ & 1(1) & $0(1)$ & $17(1)$ \\
\hline
\end{tabular}

Table 5. Hydrogen coordinates $\left(\times 10^{4}\right)$ and isotropic displacement parameters $\left(\AA^{2} \times 10\right.$ 3) for irvinylm.

$\begin{array}{lllll}\mathrm{x} & \mathrm{y} & \mathrm{z} & \mathrm{U} & \mathrm{eq}\end{array}$

$\begin{array}{lrrrr}\mathrm{H}(1 \mathrm{~A}) & 1484 & 9763 & 6020 & 85 \\ \mathrm{H}(1 \mathrm{~B}) & 2089 & 11922 & 5926 & 85 \\ \mathrm{H}(1 \mathrm{C}) & 3212 & 11364 & 6385 & 85 \\ \mathrm{H}(3) & 3148 & 12869 & 5033 & 49 \\ \mathrm{H}(5 \mathrm{~A}) & 6794 & 15288 & 4101 & 77 \\ \mathrm{H}(5 \mathrm{~B}) & 4671 & 14753 & 4232 & 77 \\ \mathrm{H}(5 \mathrm{C}) & 5110 & 13631 & 3738 & 77 \\ \mathrm{H}(6 \mathrm{~A}) & 7965 & 6955 & 6283 & 77 \\ \mathrm{H}(6 \mathrm{~B}) & 8531 & 5961 & 5778 & 77 \\ \mathrm{H}(6 \mathrm{C}) & 6365 & 5235 & 5915 & 77 \\ \mathrm{H}(8) & 9771 & 7784 & 5007 & 57 \\ \mathrm{H}(10 \mathrm{~A}) & 9920 & 8748 & 3714 & 78 \\ \mathrm{H}(10 \mathrm{~B}) & 11619 & 9616 & 4178 & 78 \\ \mathrm{H}(10 \mathrm{C}) & 11030 & 10958 & 3842 & 78 \\ \mathrm{H}(11) & 2446 & 6503 & 5070 & 54\end{array}$




$\begin{array}{lrrrr}\mathrm{H}(12) & 171 & 4356 & 4443 & 63 \\ \mathrm{H}(13) & 537 & 4770 & 3433 & 69 \\ \mathrm{H}(14) & 3274 & 7212 & 3047 & 60 \\ \mathrm{H}(15) & 5555 & 9346 & 3676 & 47 \\ \mathrm{H}(16) & 9410 & 13072 & 5302 & 41 \\ \mathrm{H}(17 \mathrm{~A}) & 7723 & 11479 & 6357 & 76 \\ \mathrm{H}(17 \mathrm{~B}) & 9738 & 13414 & 6245 & 76\end{array}$

\section{Theoretical details:}

All calculations were performed using the hybrid DFT functional B3LYP as implemented by the Jaguar 5.0 program package. ${ }^{3}$ This DFT functional utilizes the Becke three-parameter functional ${ }^{4}$ (B3) combined with the correlation functional of Lee, Yang, and $\mathrm{Par}^{5}$ (LYP), and is known to produce good descriptions of reaction profiles for transition metal containing compounds. ${ }^{6,7}$ The metals were described by the Wadt and $\mathrm{Hay}^{8}$ core-valence (relativistic) effective core potential (treating the valence electrons explicitly) using the LACVP basis set with the valence double- $\zeta$ contraction of the basis functions, LACVP**. All electrons were used for all other elements using a modified variant of Pople's ${ }^{9} 6-31 \mathrm{G}^{* *}$ basis set, where the six $d$ functions have been reduced to five.

Implicit solvent effects of the experimental benzene medium were calculated with the PoissonBoltzmann (PBF) continuum approximation, ${ }^{10}$ using the parameters $\varepsilon=2.284$ and $\mathrm{r}_{\text {solv }}=2.602 \AA$. Due to the increased cost of optimizing systems in the solvated phase (increase in computation time by a factor of $\sim 4$ ) solvation effects are calculated here as single point solvation corrections to gas phase geometries. Our previous work on the Ir.acac system has shown that the total energies, geometries, frequencies and zero point energies were also largely unchanged when the systems were optimized in the solvation phase.

All energies here are reported as $\Delta \mathrm{E}+$ zero point energy corrections at $0 \mathrm{~K}+$ solvation correction. Relative energies on the $\Delta \mathrm{H}(0 \mathrm{~K})$ surface are expected to be accurate to within $3 \mathrm{kcal} / \mathrm{mol}$ for stable intermediates, and within $5 \mathrm{kcal} / \mathrm{mol}$ for transition structures. Moreover, relative energies of iso-electronic species (such as regio-isomers) are considerably more accurate, since the errors largely cancel.

Free energies are not included, due to the inadequacies of free energy calculations in solutions. A more thorough analysis of this can be found in ref [6]. However, a free energy term is implicitly included in the PBF solvation methodology.

3 Jaguar 5.0, Schrodinger, Inc., Portland, Oregon, 2000

4 Becke, A. D. J. Chem. Phys. 1993, 98, 5648.

$5 \quad$ Lee, C.; Yang, W.; Parr, R. G. Phys. Rev. B 1988, 37, 785.

$6 \quad$ Baker, J.; Muir, M.; Andzelm, J.; Scheiner, A. In Chemical Applications of Density-Functional Theory; Laird, B. B., Ross, R. B., Ziegler, T., Eds.; ACS Symposium Series 629; American Chemical Society: Washington, DC, 1996.

$7 \quad$ Niu, S.; Hall, B. M. Chem. Rev. 2000, 100, 353.

8 a) Hay, P. J.; Wadt, W. R. J. Chem. Phys. 1985, 82, 299. b) Goddard, W. A., III Phys. Rev. 1968, 174, 659. c) Melius, C. F.; Olafson, B. O.; Goddard, W. A., III Chem. Phys. Lett. 1974, $28,457$.

9 (a) Hariharan, P. C.; Pople, J. A. Chem. Phys. Lett. 1972, 16, 217. (b) Francl, M. M.; Pietro, W. J.; Hehre, W. J.; Binkley, J. S.; Gordon, M. S.; DeFrees, D. J.; Pople, J. A. J. Chem. Phys. 1982, 77, 3654.

10 (a) Tannor, D. J.; Marten, B.; Murphy, R.; Friesner, R. A.; Sitkoff, D.; Nicholls, A.; Ringnalda, M.; Goddard, W. A., III; Honig, B. J. Am. Chem. Soc. 1994, 116, 11875. (b) Marten, B.; Kim, K.; Cortis, C.; Friesner, R. A.; Murphy, R. B.; Ringnalda, M. N.; Sitkoff, D.; Honig, B. J. Phys. Chem. 1996, 100, 11775. 
All geometries were optimized and evaluated for the correct number of imaginary frequencies through vibrational frequency calculations using the analytic Hessian. Zero imaginary frequencies correspond to a local minimum, while one imaginary frequency corresponds to a transition structure. Although the singlet states are expected to be the lowest energy spin states, we also investigated higher spin states for select geometries, and invariably found the singlet as the lowest energy state.

To reduce computational time the methyl groups on the acac ligands were replaced with hydrogens. Control calculations show that relative energies of intermediates and transition structures change less than $0.1 \mathrm{kcal} / \mathrm{mol}$ when methyl groups are included.

\begin{tabular}{|c|c|c|c|}
\hline \multicolumn{4}{|c|}{ Pyr-Ir-vin } \\
\hline \multicolumn{4}{|c|}{ Gas phase Energy: -964.19036812383 hartrees } \\
\hline \multicolumn{4}{|c|}{ Solvation Energy: -964.20006130887 hartrees } \\
\hline \multicolumn{4}{|c|}{ Zero Point Energy: 157.441 kcal/mol } \\
\hline \multicolumn{4}{|c|}{ Coordinates: } \\
\hline $\mathrm{O} 1$ & 1.5932534944 & -0.5184028539 & 1.3817330285 \\
\hline $\mathrm{C} 2$ & 1.3221968773 & -0.4946897464 & 2.6260619065 \\
\hline $\mathrm{C} 3$ & 0.0751305194 & -0.5901293671 & 3.2560498148 \\
\hline $\mathrm{C} 4$ & -1.1657778930 & -0.7438128098 & 2.6272899447 \\
\hline O5 & -1.4271424456 & -0.8135898415 & 1.3815792709 \\
\hline H6 & -2.0472551030 & -0.8264288582 & 3.2753928779 \\
\hline $\mathrm{H} 7$ & 0.0713257257 & -0.5486559472 & 4.3400161333 \\
\hline $\mathrm{H} 8$ & 2.2014692779 & -0.3954888841 & 3.2745466398 \\
\hline O9 & -1.4265292747 & -0.8136631278 & -1.3819722770 \\
\hline $\mathrm{C} 10$ & -1.1645961887 & & 5816054 \\
\hline $\mathrm{C} 11$ & 0.0764820830 & -0.58983 & 7694776 \\
\hline $\mathrm{C} 12$ & 1.3232925148 & -0.49416 & -2.6251967950 \\
\hline 013 & 1.5937836585 & -0.5 & 7704895 \\
\hline H14 & 2.2028394501 & -0.3 & 51592 \\
\hline H15 & 0.0731507518 & -0.5 & 98522 \\
\hline H16 & -2.0457093254 & -0.8 & 569583 \\
\hline $\mathrm{C} 17$ & -0.1309213992 & 1.365 & 518330 \\
\hline H18 & -1.1672100176 & 1.7187800327 & 6031979 \\
\hline H19 & 0.6465477767 & 3.3415 & 57169 \\
\hline $\mathrm{C} 22$ & 0.8544512818 & 2.2713894691 & 0.0005573484 \\
\hline $\mathrm{H} 23$ & 1.9033163817 & 1.9828028238 & 0.0010488395 \\
\hline Ir28 & 0.0824603025 & -0.64009 & 358292 \\
\hline $\mathrm{C} 29$ & 0.6019289611 & -5.6549956701 & -0.0020111258 \\
\hline $\mathrm{C} 30$ & -0.6663263088 & -5.0745263688 & -0.0030894248 \\
\hline $\mathrm{C} 31$ & -0.7700850105 & -3.6868903337 & -0.0019987754 \\
\hline N32 & 0.3098523197 & -2.8862643246 & 0.0000380802 \\
\hline C33 & 1.5328830675 & -3.4440854920 & 0.0009840435 \\
\hline C34 & 1.7210292530 & -4.8227076508 & 0.0000911097 \\
\hline H35 & 0.7160125327 & -6.7350836927 & -0.0027976525 \\
\hline H36 & -1.5650662479 & -5.6825983724 & -0.0047669936 \\
\hline H37 & -1.7294940171 & -3.1792327894 & -0.0027026849 \\
\hline H38 & 2.3650099287 & -2.7472702842 & 0.0026148919 \\
\hline H39 & 2.7269816342 & -5.2294796201 & 0.0010209291 \\
\hline
\end{tabular}

A

Gas phase Energy: -794.48479221782 hartrees

Solvation Energy: -794.49188769341 hartrees

Zero Point Energy: 135.008 kcal/mol

Coordinates: 


$\begin{array}{lccc}\text { Ir1 } & -0.3103940079 & 0.0543976600 & -0.1754715531 \\ \text { O2 } & -1.9725546137 & -0.1160782547 & -1.3771000460 \\ \text { O3 } & 0.7975380987 & 0.1964712806 & -1.9268462855 \\ \text { C4 } & 2.0612669312 & 0.3404504996 & -1.8778241799 \\ \text { C5 } & 2.8944446046 & 0.4262628872 & -0.7565016429 \\ \text { C6 } & 2.4925612696 & 0.3564785543 & 0.5803444341 \\ \text { O7 } & 1.3177096170 & 0.2220886493 & 1.0596321408 \\ \text { H8 } & 3.2774265303 & 0.4222850434 & 1.3430330705 \\ \text { H9 } & 3.9560394854 & 0.5455757920 & -0.9426695327 \\ \text { H10 } & 2.5443520220 & 0.4002561474 & -2.8611130050 \\ \text { C11 } & -0.4318464361 & 2.0962350180 & -0.2613770365 \\ \text { C12 } & -1.0360270989 & 2.7892057452 & -1.2266684092 \\ \text { H13 } & -0.9889926001 & 3.8774814433 & -1.2507496426 \\ \text { H14 } & 0.1115734781 & 2.6229770395 & 0.5273253526 \\ \text { H21 } & -1.5942767754 & 2.3055185885 & -2.0230099660 \\ \text { O22 } & 0.2134868384 & -2.0812581964 & -0.4354482488 \\ \text { C23 } & -0.4575253526 & -2.7890247238 & -1.2384687148 \\ \text { C24 } & -2.2491958187 & -1.2016916278 & -1.9940899478 \\ \text { C25 } & -1.6056602077 & -2.4393384784 & -1.9742381038 \\ \text { H26 } & -0.0962312657 & -3.8208748952 & -1.3719521705 \\ \text { H27 } & -3.1400546510 & -1.1221980330 & -2.6294149210 \\ \text { H28 } & -2.0352799640 & -3.2138522941 & -2.6006747764 \\ \text { C29 } & -1.5955242324 & 0.5580215061 & 1.5539806709 \\ \text { C30 } & -1.4023415053 & -0.8232165178 & 1.5036605271 \\ \text { H31 } & -1.0128512994 & 1.1655482359 & 2.2386558892 \\ \text { H32 } & -0.6619793590 & -1.3016797100 & 2.1371821572 \\ \text { H33 } & -2.1676598619 & -1.4723294539 & 1.0884528954 \\ \text { H34 } & -2.5034434484 & 1.0074452251 & 1.1678847708\end{array}$

TS1

Gas phase Energy: - -794.44762075943 hartrees

Solvation Energy: -794.45490767787 hartrees

Zero Point Energy: $134.645 \mathrm{kcal} / \mathrm{mol}$

Coordinates:

$\begin{array}{lrrr}\text { Ir1 } & -0.0283317435 & 0.0668765842 & 0.0209385056 \\ \text { O2 } & 0.0075469030 & -0.1078949541 & 2.0682324721 \\ \text { O3 } & 2.1385649501 & -0.2498116085 & 0.0384152831 \\ \text { C4 } & 2.7734677771 & -0.2399881067 & -1.0511808869 \\ \text { C5 } & 2.2865080545 & -0.0162864159 & -2.3564942491 \\ \text { C6 } & 0.9653682483 & 0.2069670521 & -2.7346656607 \\ \text { O7 } & -0.1005670711 & 0.2390144679 & -2.0250155383 \\ \text { H8 } & 0.7789206386 & 0.3654481092 & -3.8042626315 \\ \text { H9 } & 3.0216184879 & -0.0212732042 & -3.1545650668 \\ \text { H10 } & 3.8561019131 & -0.4273072162 & -0.9700341639 \\ \text { C11 } & -0.3560674828 & -2.0704634131 & 0.0708918361 \\ \text { H12 } & -0.2436530007 & -2.4056245621 & 1.1008216616 \\ \text { H13 } & 0.4830293251 & -3.8518916534 & -0.7352819029 \\ \text { C14 } & 0.0196830541 & -2.8880201871 & -0.9327943726 \\ \text { H15 } & -0.1167431538 & -2.6145020356 & -1.9752607583 \\ \text { O16 } & 0.5362175851 & 2.0553540472 & 0.0344582649 \\ \text { C17 } & 0.7830887416 & 2.6617166750 & 1.1251317352 \\ \text { C18 } & 0.3503469203 & 0.8738220034 & 2.8075026651 \\ \text { C19 } & 0.7148030108 & 2.1704217899 & 2.4344949694 \\ \text { H20 } & 1.0865192354 & 3.7087572206 & 0.9983035846\end{array}$




$\begin{array}{lrrc}\mathrm{H} 21 & 0.3491068287 & 0.6443152954 & 3.8799978867 \\ \mathrm{H} 22 & 0.9720261643 & 2.8578716840 & 3.2328393066 \\ \mathrm{C} 23 & -1.9991210100 & -1.2424714425 & -0.1583075985 \\ \mathrm{C} 24 & -2.0886040595 & 0.2105701232 & 0.0586697952 \\ \mathrm{H} 25 & -2.2345861732 & -1.5747941083 & -1.1646548232 \\ \mathrm{H} 26 & -2.4716692438 & 0.7883489370 & -0.7817047087 \\ \mathrm{H} 27 & -2.4799903401 & 0.5350749737 & 1.0235535114 \\ \mathrm{H} 28 & -2.4408811414 & -1.8718862253 & 0.6142347901\end{array}$

B

Gas phase Energy: - 794.50733773967 hartrees

Solvation Energy: -794.51380169718 hartrees

Zero Point Energy: $136.778 \mathrm{kcal} / \mathrm{mol}$

Coordinates:

$\begin{array}{lccc}\text { Ir1 } & -0.4982296488 & 0.1159601470 & -0.3363496583 \\ \text { O2 } & -1.2028805513 & 0.2947321421 & -2.2597885882 \\ \text { O3 } & 1.3602310126 & 0.0083783217 & -1.2454190964 \\ \text { C4 } & 2.4253081996 & -0.1830886701 & -0.5749944328 \\ \text { C5 } & 2.5704961360 & -0.3341620368 & 0.8075271591 \\ \text { C6 } & 1.5356971108 & -0.2770644602 & 1.7452780683 \\ \text { O7 } & 0.2904854833 & -0.0806486465 & 1.5547839941 \\ \text { H8 } & 1.8074761026 & -0.4061978211 & 2.7998360649 \\ \text { H9 } & 3.5726236278 & -0.5012487012 & 1.1863154258 \\ \text { H10 } & 3.3378088815 & -0.2297716626 & -1.1829104445 \\ \text { O11 } & -0.4347737776 & -2.0990579541 & -0.4325481864 \\ \text { C12 } & -0.7289349106 & -2.6679689495 & -1.5190670871 \\ \text { C13 } & -1.3459415475 & -0.7356143067 & -3.0083547971 \\ \text { C14 } & -1.1606012538 & -2.0884905004 & -2.7311292721 \\ \text { H15 } & -0.6344814149 & -3.7660118530 & -1.5221757569 \\ \text { H16 } & -1.6711440849 & -0.4851713676 & -4.0257822854 \\ \text { H17 } & -1.3549063384 & -2.7735743140 & -3.5499300605 \\ \text { C18 } & -0.5101351024 & 2.2195693750 & -0.3157227463 \\ \text { H19 } & 0.1968207165 & 2.5990943387 & 0.4285655169 \\ \text { H20 } & -0.2638170428 & 2.6027470163 & -1.3085301156 \\ \text { C21 } & -1.9737711121 & 2.4131654705 & 0.1079654996 \\ \text { H22 } & -2.1771686824 & 3.2695718814 & 0.7617961346 \\ \text { H23 } & -2.6337828843 & 2.4762983009 & -0.7631046433 \\ \text { H24 } & -2.7981619980 & -0.9783698407 & 0.7911038460 \\ \text { C25 } & -2.6503439182 & -0.0760823413 & 0.2052729829 \\ \text { C26 } & -2.1424719870 & 1.0724805918 & 0.8059759210 \\ \text { H27 } & -1.8407787839 & 1.0196050079 & 1.8499571094 \\ \text { H28 } & -3.1897723485 & -0.0152150569 & -0.7359384757\end{array}$

TS2

Gas phase Energy: - -794.47306547711 hartrees

Solvation Energy: -794.48074903713 hartrees

Zero Point Energy: $133.426 \mathrm{kcal} / \mathrm{mol}$

Coordinates:

$\begin{array}{llrc}\text { Ir1 } & -.0201549065 & -.0305340915 & -.0067083946 \\ \text { O2 } & -.1168741960 & -.0681636663 & 2.0482920125 \\ \text { O3 } & 2.0436200479 & .0423511648 & .2094688689 \\ \text { C4 } & 2.8013303079 & .0244731837 & -.8109891986 \\ \text { C5 } & 2.4488512133 & -.0009662623 & -2.1668842205 \\ \text { C6 } & 1.1503806613 & -.0123646782 & -2.6818666744\end{array}$




$\begin{array}{lccc}\text { O7 } & .0376209312 & -.0226293250 & -2.0565347868 \\ \text { H8 } & 1.0446916114 & -.0106853465 & -3.7733151065 \\ \text { H9 } & 3.2610172230 & -.0004515576 & -2.8856216298 \\ \text { H10 } & 3.8737933647 & .0368304339 & -.5763701860 \\ \text { O11 } & .2629516210 & 2.1299391454 & -.0669214665 \\ \text { C12 } & .1890395085 & 2.7997494795 & .9997568221 \\ \text { C13 } & -.1383996736 & 1.0155064807 & 2.7312541129 \\ \text { C14 } & -.0178696437 & 2.3395200724 & 2.3157471771 \\ \text { H15 } & .3022732827 & 3.8886032449 & .8808730828 \\ \text { H16 } & -.2573505051 & .8466966984 & 3.8086502674 \\ \text { H17 } & -.0569172675 & 3.0946254589 & 3.0936747281 \\ \text { C18 } & -.5453304479 & -2.0367384641 & -.0273325170 \\ \text { H19 } & -.2063401572 & -2.5873629644 & -.9036564505 \\ \text { H20 } & -.3548157683 & -2.5476234304 & .9157550358 \\ \text { C21 } & -1.8464966154 & -1.3543086601 & -.1508896948 \\ \text { H22 } & -1.6814452234 & .0432227585 & -.1272603513 \\ \text { H23 } & -2.2834309270 & -1.3617429672 & -1.1511529835 \\ \text { H24 } & -4.8541436262 & -1.6535684577 & 1.5726180150 \\ \text { C26 } & -4.1595712423 & -1.5421513966 & .7462785317 \\ \text { C27 } & -2.8444860106 & -1.4569440095 & .9509212974 \\ \text { H27 } & -2.4302889321 & -1.4803608431 & 1.9560747259 \\ \text { H28 } & -4.5876938085 & -1.5167524559 & -.2530902517\end{array}$

C

Gas phase Energy: -794.49647952368 hartrees Solvation Energy: -794.50399980482 hartrees Zero Point Energy: $134.759 \mathrm{kcal} / \mathrm{mol}$ Coordinates:

$\begin{array}{lccc}\text { O1 } & -1.6597216284 & -0.0884832435 & -1.8916745252 \\ \text { O2 } & 1.1661662009 & 0.0547910170 & -1.7912906148 \\ \text { C3 } & 2.3959923200 & 0.0972423998 & -1.4629752771 \\ \text { C4 } & 2.9645407483 & 0.1472410025 & -0.1857437155 \\ \text { C5 } & 2.2683375695 & 0.1532499362 & 1.0270431642 \\ \text { O6 } & 1.0100724861 & 0.1326657191 & 1.2281121983 \\ \text { H7 } & 2.8642196835 & 0.1822892137 & 1.9470738305 \\ \text { H8 } & 4.0471667813 & 0.1725316149 & -0.1301563412 \\ \text { H9 } & 3.0874947924 & 0.0916225558 & -2.3152033444 \\ \text { O10 } & 0.0891998710 & -2.1476355636 & -0.3977235874 \\ \text { C11 } & -0.4380174807 & -2.8518449143 & -1.3032825960 \\ \text { C12 } & -1.8656713281 & -1.1839824356 & -2.5197162324 \\ \text { C13 } & -1.3468443575 & -2.4610569299 & -2.3052516880 \\ \text { H14 } & -0.1496365271 & -3.9154243709 & -1.3034521171 \\ \text { H15 } & -2.5714989933 & -1.0770545448 & -3.3531339564 \\ \text { H16 } & -1.6803145322 & -3.2358643654 & -2.9875976517 \\ \text { H17 } & -0.3232241077 & 1.5937201626 & -0.4604689566 \\ \text { Ir18 } & -0.3143873823 & 0.0311468664 & -0.3353301468 \\ \text { C19 } & -1.8450014287 & 0.8260277958 & 1.1023362221 \\ \text { C20 } & -1.8309464799 & -0.5811569958 & 1.0770122561 \\ \text { H21 } & -1.2696069605 & 1.3259493292 & 1.8783487996 \\ \text { H22 } & -1.3208104541 & -1.1314647877 & 1.8609848768 \\ \text { H23 } & -2.6155408925 & -1.1204373923 & 0.5516281667 \\ \text { C25 } & -3.1970063822 & 2.8803926980 & 0.8364863344 \\ \text { C26 } & -2.8969751236 & 1.6313967490 & 0.4648364890 \\ \text { H27 } & -3.9975505383 & 3.4368112950 & 0.3598493684\end{array}$




$\begin{array}{lllr}\mathrm{H} 28 & -3.4489513513 & 1.1495970518 & -0.3389627233 \\ \mathrm{H} 29 & -2.6515462988 & 3.3873551654 & 1.6288168775\end{array}$

TS3

Gas phase Energy: - -794.47329653236 hartrees

Solvation Energy: -794.48092360164 hartrees

Zero Point Energy: 133.476 kcal/mol

Coordinates:

$\begin{array}{lccc}\text { Ir1 } & -0.0331766425 & -0.0305046704 & -0.0012321227 \\ \text { O2 } & -0.1303102604 & -0.1066057397 & 2.0491535723 \\ \text { O3 } & 2.0317653034 & 0.0678793301 & 0.2230927105 \\ \text { C4 } & 2.7859291693 & 0.1840765419 & -0.7959497164 \\ \text { C5 } & 2.4316528922 & 0.2494000614 & -2.1478844112 \\ \text { C6 } & 1.1340602421 & 0.1865690752 & -2.6669566060 \\ \text { O7 } & 0.0276481101 & 0.0546520580 & -2.0491217355 \\ \text { H8 } & 1.0282321949 & 0.2505624652 & -3.7563847259 \\ \text { H9 } & 3.2400712479 & 0.3574694856 & -2.8626724141 \\ \text { H10 } & 3.8568223539 & 0.2364337376 & -0.5594587521 \\ \text { O11 } & 0.2404620470 & 2.1290653508 & -0.0441629280 \\ \text { C12 } & 0.2827518290 & 2.7676446798 & 1.0449288637 \\ \text { C13 } & -0.0013815436 & 0.9530814136 & 2.7556989083 \\ \text { C14 } & 0.1798268322 & 2.2775664413 & 2.3613267183 \\ \text { H15 } & 0.4168181754 & 3.8557014653 & 0.9443675302 \\ \text { H16 } & -0.0519383244 & 0.7631701940 & 3.8349175451 \\ \text { H17 } & 0.2533004364 & 3.0104375346 & 3.1578859045 \\ \text { C18 } & -0.5387237171 & -2.0758510459 & 0.0313880419 \\ \text { H20 } & -0.3409589869 & -2.4340884612 & 1.0427103782 \\ \text { C21 } & -1.8086945211 & -1.3571537499 & -0.1205775926 \\ \text { H22 } & -1.6939440062 & 0.0438475638 & -0.0980124667 \\ \text { H23 } & -2.3035717654 & -1.4030794607 & -1.0891342002 \\ \text { H24 } & -2.4930023046 & -1.3779043206 & 0.7265073470 \\ \text { C25 } & -0.5532832835 & -3.3161024688 & -2.1753747716 \\ \text { C26 } & 0.0188123028 & -2.9516425570 & -1.0223088675 \\ \text { H27 } & -0.0322568596 & -3.9559433185 & -2.8809522150 \\ \text { H28 } & 1.0170731992 & -3.3280231703 & -0.7976138237 \\ \text { H29 } & -1.5522836052 & -2.9985351886 & -2.4612352953 \\ & & & \end{array}$

D

Gas phase Energy: - 794.47424569024 hartrees

Solvation Energy: -794.48195182986 hartrees

Coordinates:

$\begin{array}{lccc}\text { Ir1 } & .0017247274 & -.0007887078 & .0001303947 \\ \text { O2 } & .0016244369 & .0000423168 & 2.0540366155 \\ \text { O3 } & 2.0341716306 & .0013444229 & .1333917120 \\ \text { C4 } & 2.7555777478 & .0410745789 & -.9197699044 \\ \text { C5 } & 2.3558223843 & .0750161723 & -2.2568112698 \\ \text { C6 } & 1.0345699115 & .0673914160 & -2.7213978745 \\ \text { O7 } & -.0437080411 & .0179754781 & -2.0470370779 \\ \text { H8 } & .8832707487 & .1025824084 & -3.8065152915 \\ \text { H9 } & 3.1405723740 & .1135072769 & -3.0042082288 \\ \text { H10 } & 3.8325338120 & .0464354375 & -.7143201538 \\ \text { O11 } & .3683686897 & 2.1665132032 & -.1278366963 \\ \text { C12 } & .5226232610 & 2.8248054644 & .9373477988 \\ \text { C13 } & .2433014863 & 1.0706363434 & 2.7154585695\end{array}$




$\begin{array}{cccc}\text { C14 } & .4833443145 & 2.3690593112 & 2.2713546057 \\ \text { H15 } & .7094536466 & 3.9023585452 & .8041885448 \\ \text { H16 } & .2473498775 & .9153090621 & 3.8014477377 \\ \text { H17 } & .6545921565 & 3.1142257089 & 3.0412490115 \\ \text { C18 } & -.7129511938 & -1.9490977753 & .1250114708 \\ \text { H19 } & -.5257030997 & -2.3094104308 & 1.1388560988 \\ \text { C20 } & -1.9753730263 & -1.1522269951 & .0052400381 \\ \text { H21 } & -1.7788019466 & .0652865321 & -.0263118208 \\ \text { H22 } & -2.5217839495 & -1.2579014228 & -.9316543062 \\ \text { H23 } & -2.6247099368 & -1.1723470895 & .8815495079 \\ \text { C24 } & -.9564595656 & -3.2581187644 & -2.0369147468 \\ \text { C25 } & -.3172865190 & -2.9336289821 & -.9069561656 \\ \text { H26 } & -.5410464375 & -3.9891590471 & -2.7237073298 \\ \text { H27 } & .6263562398 & -3.4346008322 & -.6877965236 \\ \text { H28 } & -1.9072165357 & -2.8174171838 & -2.3240594943 \\ \text { TS4 } & & & \end{array}$

Gas phase Energy: - 794.47424569024 hartrees

Solvation Energy: -794.48195182986 hartrees

Coordinates:

$\begin{array}{lccc}\text { Ir1 } & .0017247274 & -.0007887078 & .0001303947 \\ \text { O2 } & .0016244369 & .0000423168 & 2.0540366155 \\ \text { O3 } & 2.0341716306 & .0013444229 & .1333917120 \\ \text { C4 } & 2.7555777478 & .0410745789 & -.9197699044 \\ \text { C5 } & 2.3558223843 & .0750161723 & -2.2568112698 \\ \text { C6 } & 1.0345699115 & .0673914160 & -2.7213978745 \\ \text { O7 } & -.0437080411 & .0179754781 & -2.0470370779 \\ \text { H8 } & .8832707487 & .1025824084 & -3.8065152915 \\ \text { H9 } & 3.1405723740 & .1135072769 & -3.0042082288 \\ \text { H10 } & 3.8325338120 & .0464354375 & -.7143201538 \\ \text { O11 } & .3683686897 & 2.1665132032 & -.1278366963 \\ \text { C12 } & .5226232610 & 2.8248054644 & .9373477988 \\ \text { C13 } & .2433014863 & 1.0706363434 & 2.7154585695 \\ \text { C14 } & .4833443145 & 2.3690593112 & 2.2713546057 \\ \text { H15 } & .7094536466 & 3.9023585452 & .8041885448 \\ \text { H16 } & .2473498775 & .9153090621 & 3.8014477377 \\ \text { H17 } & .6545921565 & 3.1142257089 & 3.0412490115 \\ \text { C18 } & -.7129511938 & -1.9490977753 & .1250114708 \\ \text { H19 } & -.5257030997 & -2.3094104308 & 1.1388560988 \\ \text { C20 } & -1.9753730263 & -1.1522269951 & .0052400381 \\ \text { H21 } & -1.7788019466 & .0652865321 & -.0263118208 \\ \text { H22 } & -2.5217839495 & -1.2579014228 & -.9316543062 \\ \text { H23 } & -2.6247099368 & -1.1723470895 & .8815495079 \\ \text { C24 } & -.9564595656 & -3.2581187644 & -2.0369147468 \\ \text { C25 } & -.3172865190 & -2.9336289821 & -.9069561656 \\ \text { H26 } & -.5410464375 & -3.9891590471 & -2.7237073298 \\ \text { H27 } & .6263562398 & -3.4346008322 & -.6877965236 \\ \text { H28 } & -1.9072165357 & -2.8174171838 & -2.3240594943 \\ & & & \end{array}$

$\mathrm{E}$

Gas phase Energy: - 794.52048270447 hartrees

Solvation Energy: -794.52684381786 hartrees

Zero Point Energy: $136.471 \mathrm{kcal} / \mathrm{mol}$

Coordinates:

$\begin{array}{llll}\text { O1 } & -1.7696796210 & -0.0061785763 & -1.7800226996\end{array}$ 


$\begin{array}{lccc}\text { O2 } & 1.0513273678 & -0.0111191359 & -1.8668447404 \\ \text { C3 } & 2.2931339993 & -0.0393838752 & -1.6214966894 \\ \text { C4 } & 2.9416241268 & 0.0423970266 & -0.3774923888 \\ \text { C5 } & 2.3299883002 & 0.1690173492 & 0.8694425405 \\ \text { O6 } & 1.0895929446 & 0.2652441562 & 1.1679830694 \\ \text { H7 } & 2.9909819759 & 0.1973959695 & 1.7442175486 \\ \text { H8 } & 4.0248130303 & -0.0145972786 & -0.3843401559 \\ \text { H9 } & 2.9388034947 & -0.1435448848 & -2.5055667115 \\ \text { O10 } & -0.0361418354 & -1.9361040374 & -0.2003153624 \\ \text { C11 } & -0.6440943596 & -2.7220684428 & -0.9855099791 \\ \text { C12 } & -2.0448545398 & -1.1362858594 & -2.3097554208 \\ \text { C13 } & -1.5729081774 & -2.4091629402 & -1.9907422935 \\ \text { H14 } & -0.4038372481 & -3.7866415400 & -0.8513171868 \\ \text { H15 } & -2.7736248034 & -1.0679986054 & -3.1266056899 \\ \text { H16 } & -1.9671027726 & -3.2359012265 & -2.5715575019 \\ \text { Ir18 } & -0.3673382936 & 0.1679757760 & -0.2797497683 \\ \text { C18 } & -0.5356685043 & 2.3290047273 & -0.0072594595 \\ \text { H19 } & 0.2131537765 & 2.5370941663 & 0.7568252470 \\ \text { C24 } & -1.6921196785 & 0.6072316876 & 1.3644229992 \\ \text { C25 } & -1.7311319397 & 1.6666879359 & 0.4042199695 \\ \text { H26 } & -2.5935601294 & 0.0158953425 & 1.4993172980 \\ \text { H27 } & -2.5995122636 & 1.7362193413 & -0.2492613382 \\ \text { H28 } & -1.0500334111 & 0.6805944785 & 2.2388857706 \\ \text { C28 } & -0.5345020445 & 3.2609227770 & -1.1947767194 \\ \text { H29 } & -0.7709209920 & 4.2898372999 & -0.8920200468 \\ \text { H30 } & 0.4499317733 & 3.2767473786 & -1.6720609219 \\ \text { H31 } & -1.2645446134 & 2.9457994468 & -1.9457874176\end{array}$

$\mathrm{F}$

Gas phase Energy: -794.51993893133 hartrees

Solvation Energy: -794.52660256246 hartrees

Zero Point Energy: $136.451 \mathrm{kcal} / \mathrm{mol}$

Coordinates:

$\begin{array}{lccc}\text { O1 } & -0.0352257428 & -0.1479168749 & 0.0702125389 \\ \text { O2 } & 0.1138280634 & 0.6505605045 & 2.7670224884 \\ \text { C3 } & 0.4303458865 & 0.8996404407 & 3.9669819984 \\ \text { C4 } & 1.6033145098 & 0.5429310670 & 4.6541834105 \\ \text { C5 } & 2.6840683364 & -0.1652711568 & 4.1304345080 \\ \text { O6 } & 2.8479051997 & -0.6631622229 & 2.9641026047 \\ \text { H7 } & 3.5364147206 & -0.3372610104 & 4.7991682193 \\ \text { H8 } & 1.6856957272 & 0.8649566277 & 5.6860741232 \\ \text { H9 } & -0.3176419535 & 1.4744070908 & 4.5335670335 \\ \text { O10 } & 2.2439906362 & 1.5031666426 & 1.2160152689 \\ \text { C11 } & 1.7233694632 & 2.3175708711 & 0.3976194937 \\ \text { C12 } & -0.1724898862 & 0.9772811913 & -0.5201916617 \\ \text { C13 } & 0.5972992068 & 2.1364226612 & -0.4217253320 \\ \text { H14 } & 2.2300221181 & 3.2910307362 & 0.3280674876 \\ \text { H15 } & -1.0223262157 & 1.0036695635 & -1.2131654510 \\ \text { H16 } & 0.3000817356 & 2.9744965389 & -1.0426315915 \\ \text { Ir17 } & 1.4249258619 & -0.4508282898 & 1.4944656881 \\ \text { C18 } & 0.9582639468 & -2.5287349816 & 1.8554068696 \\ \text { C19 } & 1.5241226023 & -2.3678463957 & 0.5512409343 \\ \text { H20 } & -0.0838454585 & -2.8294901101 & 1.9170968266 \\ \text { H21 } & 1.5846575107 & -2.8429485179 & 2.6873506324\end{array}$

S18 


$\begin{array}{lllr}\text { C22 } & 2.7898830031 & -1.7311578711 & 0.3780414630 \\ \text { H23 } & 0.8561165587 & -2.4194355991 & -0.3073556489 \\ \text { H24 } & 3.5636576787 & -1.9368072015 & 1.1172924973 \\ \text { C25 } & 3.2920813990 & -1.3491549899 & -0.9926068803 \\ \text { H26 } & 3.9330500636 & -0.4638820457 & -0.9344691776 \\ \text { H27 } & 3.8887494570 & -2.1574366737 & -1.4344269884 \\ \text { H28 } & 2.4659346286 & -1.1259104892 & -1.6734495438\end{array}$

G

Gas phase Energy: -873.11624481756 hartrees

Solvation Energy: -873.12249846053 hartrees

Zero Point Energy: $170.267 \mathrm{kcal} / \mathrm{mol}$

Coordinates:

$\begin{array}{cccc}\text { O1 } & -1.0870548907 & 0.2268945943 & -2.1895981160 \\ \text { O2 } & 1.4432922526 & -0.2644711789 & -1.5268677458 \\ \text { C3 } & 2.6098584446 & -0.5137529971 & -1.0861830923 \\ \text { C4 } & 3.0325414173 & -0.5811197316 & 0.2437844882 \\ \text { C5 } & 2.2056352767 & -0.3818158048 & 1.3510131522 \\ \text { O6 } & 0.9620329604 & -0.1038644882 & 1.3895656494 \\ \text { H7 } & 2.6657459390 & -0.4705918045 & 2.3425510704 \\ \text { H8 } & 4.0753905417 & -0.8100764750 & 0.4307401852 \\ \text { H9 } & 3.3573662193 & -0.6951373406 & -1.8682858852 \\ \text { O10 } & -0.5374367548 & -2.1212384498 & -0.2731756221 \\ \text { C11 } & -1.0281866429 & -2.7115796241 & -1.2726498562 \\ \text { C12 } & -1.4475543268 & -0.8083069790 & -2.8503181796 \\ \text { C13 } & -1.4605445490 & -2.1543151907 & -2.4941157301 \\ \text { H14 } & -1.1325013472 & -3.8052877585 & -1.1806306160 \\ \text { H15 } & -1.8158103692 & -0.5643587740 & -3.8549042273 \\ \text { H16 } & -1.8380769387 & -2.8460440988 & -3.2398491914 \\ \text { Ir17 } & -0.1979264895 & 0.0865838394 & -0.3221438967 \\ \text { C18 } & -1.6418027658 & 0.2274193214 & 1.3463131888 \\ \text { C19 } & -2.2973975982 & 0.6094735626 & 0.1850651630 \\ \text { H20 } & -1.7383900710 & -0.7825227799 & 1.7323784303 \\ \text { H21 } & -2.9268717364 & -0.0969757642 & -0.3472105783 \\ \text { H22 } & -2.4276173644 & 1.6531417327 & -0.0798932549 \\ \text { H23 } & -1.2442805330 & 0.9647355578 & 2.0355292257 \\ \text { C25 } & 0.2818517461 & 2.1353132297 & -0.2909973364 \\ \text { H26 } & 1.3787760854 & 2.1507985092 & -0.2388651220 \\ \text { H27 } & -0.0993237091 & 2.5792212983 & 0.6378339583 \\ \text { C28 } & -1.0711734979 & 3.9094537832 & -1.4569523200 \\ \text { C29 } & -0.1884370759 & 2.9013394358 & -1.4760756560 \\ \text { H30 } & 0.2212550236 & 2.5907557545 & -2.4379252674 \\ \text { H31 } & -1.4847915404 & 4.2280657052 & -0.4972270505 \\ \text { C32 } & -1.5399690471 & 4.6672463377 & -2.6673154162 \\ \text { H33 } & -2.6283711425 & 4.5913178256 & -2.7949890868 \\ \text { H34 } & -1.3100474701 & 5.7384913957 & -2.5889434430 \\ \text { H35 } & -1.0693279189 & 4.2880473291 & -3.5801488965 \\ \text { TS5 } & & & \\ \text { G } 5 h a & & & \end{array}$

Gas phase Energy: -873.06319169335 hartrees

Solvation Energy: -873.07020307424 hartrees

Zero Point Energy: $166.705 \mathrm{kcal} / \mathrm{mol}$

Coordinates:
Ir1
$\begin{array}{ll}0.0079180924 & 0.0561288279\end{array}$
0.0773586184
$\begin{array}{llll}\mathrm{O} 2 & -0.0877690132 & 0.5070131981 & 2.1508434532\end{array}$ 


$\begin{array}{lrrr}\text { C3 } & 0.9671671296 & 0.5059420141 & 2.8579074213 \\ \text { C4 } & 2.2866302011 & 0.2432819565 & 2.4674675332 \\ \text { C5 } & 2.7171526812 & -0.1016238588 & 1.1849164582 \\ \text { O6 } & 2.0394299248 & -0.2630680852 & 0.1155177257 \\ \text { H7 } & 3.7923897675 & -0.2709040499 & 1.0508706542 \\ \text { H8 } & 3.0486405231 & 0.3106392006 & 3.2361102728 \\ \text { H9 } & 0.1673125379 & 1.3212312940 & -2.1211909462 \\ \text { O10 } & -2.0233914020 & 0.3870232714 & 0.0483811368 \\ \text { O11 } & 0.4705759836 & 2.1919106057 & 0.0051750368 \\ \text { C12 } & -0.4734629383 & 3.0358340214 & 0.0596204112 \\ \text { C13 } & -2.5071264101 & 1.5685795320 & 0.0891422690 \\ \text { C14 } & -1.8587454047 & 2.8056361283 & 0.0969416561 \\ \text { H15 } & -0.1581099581 & 4.0902229026 & 0.0697639138 \\ \text { H16 } & -3.6035087165 & 1.5877091428 & 0.1174670805 \\ \text { H17 } & -2.4959820288 & 3.6825837903 & 0.1371622503 \\ \text { C18 } & -0.4106397845 & -1.8787916855 & 0.6528525176 \\ \text { H19 } & -0.8826563766 & -0.0368809578 & -2.5934338442 \\ \text { H23 } & -1.4347792907 & -1.9742633876 & 1.0164058111 \\ \text { H24 } & 0.1081778026 & -3.8749364791 & 1.0995849753 \\ \text { C25 } & 0.4217664402 & -2.9199424989 & 0.6810349710 \\ \text { H26 } & 1.4379568117 & -2.8645135037 & 0.3005048061 \\ \text { H27 } & 0.8067983737 & 0.7503989597 & 3.9170314356 \\ \text { H28 } & -0.1500109900 & -1.0006463233 & -1.0657860089 \\ \text { C29 } & 0.0779592392 & 0.2338594019 & -2.1469610012 \\ \text { C27 } & 1.1334925560 & -1.2852510673 & -3.8346456406 \\ \text { C28 } & 1.2353948865 & -0.3920823237 & -2.8428668965 \\ \text { H30 } & 2.2206702162 & -0.0856118509 & -2.4969278130 \\ \text { H31 } & 0.1394471923 & -1.5953287748 & -4.1632426169 \\ \text { C31 } & 2.2949149104 & -1.9086108258 & -4.5525110844 \\ \text { H32 } & 2.2810604263 & -3.0017191261 & -4.4531586463 \\ \text { H33 } & 2.2638732884 & -1.6924736227 & -5.6282957551 \\ \text { H34 } & 3.2496378934 & -1.5451942286 & -4.1606422983\end{array}$

$\mathrm{H}$

Gas phase Energy: -873.08119925835 hartrees Solvation Energy: -873.08936312143 hartrees Zero Point Energy: $168.539 \mathrm{kcal} / \mathrm{mol}$ Coordinates:

$\begin{array}{lrrr}\text { Ir1 } & -1.2302471244 & 0.3873951195 & 0.0312090639 \\ \text { H2 } & 0.5401185862 & 0.9517206460 & 0.1874426914 \\ \text { O3 } & -1.5765425642 & 0.3693649624 & -2.1593097558 \\ \text { O4 } & -0.7232907649 & -1.5884694051 & -0.1143929703 \\ \text { H5 } & -1.3177237432 & 0.9825114519 & 4.0087905965 \\ \text { C6 } & -1.5083978121 & 1.1120439796 & 2.9437636885 \\ \text { C7 } & -0.9541384676 & 0.3047291788 & 2.0343750186 \\ \text { H8 } & -0.3015144584 & -0.5055241914 & 2.3777842032 \\ \text { H9 } & -2.1641756247 & 1.9319305837 & 2.6625771227 \\ \text { C10 } & 1.2897904763 & 0.7755552124 & -0.6691063466 \\ \text { H11 } & 0.9984734544 & -0.0359352749 & -1.3352659717 \\ \text { H12 } & 2.1472896170 & 0.4439905982 & -0.0740840296 \\ \text { C13 } & -3.6655224858 & -1.1093089258 & 0.2767931624 \\ \text { O14 } & -3.1931227308 & 0.0805426566 & 0.2544642174 \\ \text { H15 } & -4.7527801856 & -1.1336065070 & 0.4031943536 \\ \text { C16 } & -2.1210263370 & 3.0530618720 & -0.8506280243\end{array}$




$\begin{array}{llll}\text { O17 } & -1.6828024062 & 2.3921152171 & 0.1520963145 \\ \text { H18 } & -2.3702713326 & 4.0933136593 & -0.6061243490 \\ \text { C19 } & -2.0412411938 & 1.3949584843 & -2.7325281933 \\ \text { C20 } & -2.3129883843 & 2.6602811174 & -2.1766357477 \\ \text { H21 } & -2.2508476378 & 1.2865505210 & -3.8090313425 \\ \text { H22 } & -2.7072103492 & 3.4146772270 & -2.8494732968 \\ \text { C23 } & -1.6187360059 & -2.4905569564 & -0.0385748341 \\ \text { C24 } & -2.9967518011 & -2.3265956936 & 0.1547458714 \\ \text { H25 } & -1.2405380760 & -3.5135537861 & -0.1459465084 \\ \text { H26 } & -3.6012852100 & -3.2256538554 & 0.1951326108 \\ \text { C27 } & 1.5312811577 & 2.0693665619 & -1.3808804158 \\ \text { H28 } & 0.7576867243 & 2.3866015789 & -2.0782686055 \\ \text { H29 } & 3.3796347844 & 2.5036179354 & -0.4989736551 \\ \text { C30 } & 2.6152869599 & 2.8305288971 & -1.2063955655 \\ \text { C31 } & 2.8806286753 & 4.1235499556 & -1.9205710292 \\ \text { H32 } & 2.9982765902 & 4.9497713576 & -1.2079707390 \\ \text { H33 } & 2.0662906889 & 4.3789035404 & -2.6052849208 \\ \text { H34 } & 3.8113606002 & 4.0744463569 & -2.5000538648\end{array}$

I

Gas phase Energy: -638.43643103137 hartrees

Solvation Energy: -638.45223213412 hartrees

Zero Point Energy: $79.018 \mathrm{kcal} / \mathrm{mol}$

Coordinates:

$\begin{array}{lrrr}\text { Ir1 } & -0.1787342336 & 0.0660233745 & -0.1990006937 \\ \text { O2 } & 1.2461377303 & 0.2858877202 & -1.6467542170 \\ \text { O3 } & 1.2043586726 & 0.1340779749 & 1.2068592607 \\ \text { C4 } & 0.8838636026 & 0.0235019344 & 2.4485847291 \\ \text { C5 } & -0.3662147250 & -0.1375861759 & 3.0297612524 \\ \text { C6 } & -1.5806640814 & -0.2356431006 & 2.3288627871 \\ \text { O7 } & -1.7478608323 & -0.1734402719 & 1.0729320469 \\ \text { H8 } & -2.4976304339 & -0.3869096443 & 2.9088866077 \\ \text { H9 } & -0.4034082815 & -0.2134822776 & 4.1103700036 \\ \text { H10 } & 1.7610105820 & 0.0645697487 & 3.1009827041 \\ \text { H11 } & -0.3204679312 & 1.6273100369 & -0.1097394152 \\ \text { O12 } & -0.0599368651 & -2.1337040270 & -0.3431962535 \\ \text { C13 } & 0.8283521020 & -2.6853999075 & -1.0531637513 \\ \text { C14 } & 1.8998850348 & -0.7021420185 & -2.1389285086 \\ \text { C15 } & 1.7774653657 & -2.0681050363 & -1.8908841125 \\ \text { H16 } & 0.8514363345 & -3.7863550077 & -1.0209071315 \\ \text { H17 } & 2.6572593532 & -0.3970799559 & -2.8708163855 \\ \text { H18 } & 2.4599380330 & -2.7213514714 & -2.4243724706\end{array}$

$\mathrm{J}$

Gas phase Energy: -157.22950679500 hartrees

Solvation Energy: -157.23054244125 hartrees

Zero Point Energy: $68.192 \mathrm{kcal} / \mathrm{mol}$

Coordinates:

$\begin{array}{llll}\text { C1 } & 4.7416285876 & -1.0875753419 & 2.0821080269 \\ \text { C2 } & 3.5977548713 & -1.2500673604 & 1.4165618077 \\ \text { H3 } & 5.1325819198 & -1.8597773612 & 2.7411413892 \\ \text { H5 } & 3.2445327190 & -0.4446182767 & 0.7697408345 \\ \text { H6 } & 5.3320913967 & -0.1802288806 & 1.9927245699 \\ \text { C6 } & 2.7123006619 & -2.4648135566 & 1.4852686456\end{array}$




$\begin{array}{lccc}\text { H8 } & 2.6343187019 & -2.9123710629 & 0.4837249938 \\ \text { H9 } & 3.1757582870 & -3.2216991791 & 2.1294725626 \\ \text { C9 } & 1.2962803417 & -2.1408317379 & 1.9916696571 \\ \text { H10 } & 1.3271629645 & -1.7369193490 & 3.0086728252 \\ \text { H11 } & 0.8084881888 & -1.3955915972 & 1.3531642715 \\ \text { H12 } & 0.6663891063 & -3.0363643699 & 1.9994661816\end{array}$

$\mathrm{K}$

Gas phase Energy: - 717.09664277054 hartrees

Solvation Energy: -717.10356132267 hartrees

Zero Point Energy: $114.029 \mathrm{kcal} / \mathrm{mol}$

Coordinates:

$\begin{array}{lrrr}\text { Ir1 } & -0.2047533239 & 0.0903260046 & -0.2603436950 \\ \text { O2 } & 1.3240657175 & 0.2790187341 & -1.6233677476 \\ \text { O3 } & 1.2658725708 & -0.0075881268 & 1.2045796763 \\ \text { C4 } & 0.9514077358 & -0.1634915706 & 2.4288053194 \\ \text { C5 } & -0.3191508968 & -0.2772265547 & 3.0028823079 \\ \text { C6 } & -1.5386293220 & -0.2487277599 & 2.3186992108 \\ \text { O7 } & -1.7542650981 & -0.1017893987 & 1.0713543629 \\ \text { H8 } & -2.4509494421 & -0.3648530686 & 2.9158160924 \\ \text { H9 } & -0.3626162183 & -0.4112051401 & 4.0780525743 \\ \text { H10 } & 1.8108336445 & -0.2143685613 & 3.1095412544 \\ \text { H11 } & -0.1744078971 & 1.6451132378 & -0.0423586092 \\ \text { O12 } & 0.0015277384 & -2.1052008279 & -0.1781919180 \\ \text { C13 } & 0.9330828616 & -2.6646574421 & -0.8221435026 \\ \text { C14 } & 2.0087866451 & -0.7308569539 & -2.0063681055 \\ \text { C15 } & 1.8828904868 & -2.0825014992 & -1.6824879891 \\ \text { H16 } & 1.0039074444 & -3.7566906744 & -0.6946585700 \\ \text { H17 } & 2.8137543486 & -0.4672207502 & -2.7037639767 \\ \text { H18 } & 2.5994522260 & -2.7555180464 & -2.1413398242 \\ \text { C19 } & -1.6111906836 & -0.3827531655 & -1.8464734877 \\ \text { C20 } & -1.6069979047 & 1.0077284226 & -1.6760502173 \\ \text { H21 } & -1.0631336913 & -0.8375963508 & -2.6665461434 \\ \text { H22 } & -1.0472115531 & 1.6432774426 & -2.3544776495 \\ \text { H23 } & -2.3963684801 & 1.4919802152 & -1.1105842600 \\ \text { H24 } & -2.3982642439 & -0.9871157035 & -1.4062944455\end{array}$

TS6

Gas phase Energy: -717.07684152860 hartrees

Solvation Energy: - 717.08419617157 hartrees

1 vibrational frequencies below $10.0 \mathrm{~cm}-1$ not included in zero-point

Zero Point Energy: $112.768 \mathrm{kcal} / \mathrm{mol}$

Coordinates:

$\begin{array}{lccc}\text { Ir1 } & -0.0251897599 & -0.0288308336 & 0.0087232469 \\ \text { O2 } & -0.1189377191 & -0.0705160328 & 2.0590723351 \\ \text { O3 } & 2.0350246341 & 0.1170323215 & 0.2203960748 \\ \text { C4 } & 2.7870200612 & 0.2062048652 & -0.8013950036 \\ \text { C5 } & 2.4309007056 & 0.2168712330 & -2.1559796001 \\ \text { C6 } & 1.1348307607 & 0.1117695050 & -2.6684074320 \\ \text { O7 } & 0.0307912279 & -0.0170028628 & -2.0417871834 \\ \text { H8 } & 1.0246419381 & 0.1326135499 & -3.7592753260 \\ \text { H9 } & 3.2365352404 & 0.3115050808 & -2.8756902458 \\ \text { H10 } & 3.8570667002 & 0.2822496654 & -0.5677649326 \\ \text { O11 } & 0.1975337835 & 2.1365231889 & -0.0615692513\end{array}$




$\begin{array}{lrrr}\text { C12 } & 0.2331998015 & 2.7946027471 & 1.0157677095 \\ \text { C13 } & -0.0105656665 & 1.0008007865 & 2.7516153192 \\ \text { C14 } & 0.1449972053 & 2.3233213744 & 2.3403088977 \\ \text { H15 } & 0.3467512978 & 3.8832762863 & 0.8969531259 \\ \text { H16 } & -0.0569476440 & 0.8231234259 & 3.8331290412 \\ \text { H17 } & 0.2069690907 & 3.0684272206 & 3.1263431676 \\ \text { C18 } & -0.4570179070 & -2.0652185799 & 0.0107218037 \\ \text { H19 } & -0.2147475043 & -2.5470557363 & 0.9553939262 \\ \text { C20 } & -1.7654071484 & -1.4139320900 & -0.0858330117 \\ \text { H21 } & -1.6949217288 & -0.0166947489 & -0.0785435955 \\ \text { H22 } & -2.2898273656 & -1.4723914636 & -1.0386831362 \\ \text { H23 } & -2.4195978230 & -1.4609075159 & 0.7838548584 \\ \text { H24 } & -0.1042859040 & -2.5989287054 & -0.8697906062\end{array}$

L

Gas phase Energy: -717.06779016891 hartrees

Solvation Energy: -717.07628998014 hartrees

Zero Point Energy: $114.300 \mathrm{kcal} / \mathrm{mol}$

Coordinates:

$\begin{array}{lrrr}\text { Ir1 } & -0.1819406049 & 0.0817567275 & -0.1775451784 \\ \text { O2 } & 1.2428335913 & 0.3257322036 & -1.6238654482 \\ \text { O3 } & 1.2345521609 & 0.0531153707 & 1.1965452934 \\ \text { C4 } & 0.9362675334 & -0.1039771104 & 2.4386952056 \\ \text { C5 } & -0.3084013944 & -0.2523260125 & 3.0375423575 \\ \text { C6 } & -1.5413654831 & -0.2682057310 & 2.3639011490 \\ \text { O7 } & -1.7363179361 & -0.1436397212 & 1.1151373112 \\ \text { H8 } & -2.4496470417 & -0.4050860146 & 2.9610388857 \\ \text { H9 } & -0.3262011021 & -0.3743953207 & 4.1145682207 \\ \text { H10 } & 1.8252986815 & -0.1187403159 & 3.0759969617 \\ \text { O11 } & -0.0779767718 & -2.1352487687 & -0.3531450651 \\ \text { C12 } & 0.7875720011 & -2.6669693727 & -1.1019171409 \\ \text { C13 } & 1.8723323411 & -0.6617789950 & -2.1492920073 \\ \text { C14 } & 1.7277200847 & -2.0308879311 & -1.9400932862 \\ \text { H15 } & 0.8034199153 & -3.7688347726 & -1.1108103787 \\ \text { H16 } & 2.6292497373 & -0.3513688931 & -2.8797080528 \\ \text { H17 } & 2.3905317757 & -2.6769251649 & -2.5064050507 \\ \text { C25 } & -0.4042908404 & 2.1295394807 & -0.1276972908 \\ \text { H26 } & 0.5356595561 & 2.5949667011 & -0.4450488537 \\ \text { H27 } & -0.6380661526 & 2.4801423560 & 0.8856056029 \\ \text { C28 } & -1.5400005875 & 2.4807374773 & -1.0955849434 \\ \text { H29 } & -2.4993395615 & 2.0660416712 & -0.7634094671 \\ \text { H30 } & -1.6724311615 & 3.5679124879 & -1.1883469294 \\ \text { H31 } & -1.3391173092 & 2.1073786133 & -2.1096226732\end{array}$

M

Gas phase Energy: -795.71973958034 hartrees

Solvation Energy: -795.72580056208 hartrees

Zero Point Energy: 149.913 kcal/mol

Coordinates:

$\begin{array}{lrrr}\text { Ir1 } & -0.1960300013 & 0.1085069433 & -0.2358498126 \\ \text { O2 } & 1.4389577523 & 0.2918767352 & -1.4748840562 \\ \text { O3 } & 1.1569200119 & -0.1514511833 & 1.3242559463 \\ \text { C4 } & 0.7409718943 & -0.3942984523 & 2.5045830263 \\ \text { C5 } & -0.5744129128 & -0.4823276162 & 2.9719237441\end{array}$




$\begin{array}{lrrr}\text { C6 } & -1.7365994446 & -0.3295765883 & 2.2064664626 \\ \text { O7 } & -1.8485566791 & -0.0820305020 & 0.9615508839 \\ \text { H8 } & -2.6959667317 & -0.4327582609 & 2.7272005195 \\ \text { H9 } & -0.7073674754 & -0.6954397396 & 4.0267842076 \\ \text { H10 } & 1.5405395231 & -0.5455341885 & 3.2410019903 \\ \text { O11 } & -0.0527116228 & -2.1158164281 & -0.2488335113 \\ \text { C12 } & 0.9116155515 & -2.6732668167 & -0.8439038596 \\ \text { C13 } & 2.1194911849 & -0.7209219336 & -1.8576427420 \\ \text { C14 } & 1.9345834634 & -2.0814153046 & -1.6092493524 \\ \text { H15 } & 0.9500632828 & -3.7708809859 & -0.7560649962 \\ \text { H16 } & 2.9793421396 & -0.4513163080 & -2.4839323333 \\ \text { H17 } & 2.6648981229 & -2.7535128276 & -2.0473135027 \\ \text { C18 } & -1.4350652914 & -0.3679151222 & -1.9602269606 \\ \text { C19 } & -1.4984584271 & 1.0123524969 & -1.7511340018 \\ \text { H20 } & -0.7925643612 & -0.7836096383 & -2.7311391389 \\ \text { H21 } & -0.9107128084 & 1.6898002731 & -2.3614741602 \\ \text { H22 } & -2.3557702171 & 1.4437076820 & -1.2449750690 \\ \text { H23 } & -2.2296000015 & -1.0160452835 & -1.6034674890 \\ \text { C25 } & -0.0924314608 & 2.1553993941 & 0.3083251307 \\ \text { H26 } & 0.1522071613 & 2.1105173494 & 1.3761029370 \\ \text { H27 } & -1.0819426924 & 2.6155010679 & 0.2288620423 \\ \text { C28 } & 0.9583287448 & 2.9869895444 & -0.4190044329 \\ \text { H29 } & 0.7623436732 & 3.0607048418 & -1.4938971546 \\ \text { H30 } & 0.9809382352 & 4.0099502759 & -0.0183037852 \\ \text { H31 } & 1.9568775896 & 2.5574549367 & -0.3021513726\end{array}$

TS7

Gas phase Energy: -795.66480186178 hartrees

Solvation Energy: -795.67154894284 hartrees

Zero Point Energy: $149.377 \mathrm{kcal} / \mathrm{mol}$

Coordinates:

$\begin{array}{lccc}\text { Ir1 } & 0.0549724313 & 0.0783836314 & 0.0257943202 \\ \text { O2 } & -0.1989392638 & 0.1490378237 & 2.0565892613 \\ \text { O3 } & 2.2266420681 & 0.0196233619 & 0.3901843473 \\ \text { C4 } & 3.0218577944 & 0.0834471570 & -0.5911630659 \\ \text { C5 } & 2.7201198287 & 0.1562017874 & -1.9648318579 \\ \text { C6 } & 1.4551583876 & 0.1269263661 & -2.5529816806 \\ \text { O7 } & 0.2979699574 & 0.0254852128 & -2.0159440921 \\ \text { H8 } & 1.4227434234 & 0.1769422308 & -3.6487614766 \\ \text { H9 } & 3.5636770795 & 0.2259530278 & -2.6437121317 \\ \text { H10 } & 4.0931270401 & 0.0776023439 & -0.3344320695 \\ \text { O11 } & 0.3557933890 & 2.0871920183 & -0.1883213196 \\ \text { C12 } & 0.3531717544 & 2.8566040497 & 0.8277850737 \\ \text { C13 } & -0.0900918917 & 1.2535429532 & 2.6862932157 \\ \text { C14 } & 0.1589120596 & 2.5309142973 & 2.1750201139 \\ \text { H15 } & 0.5279562283 & 3.9115454326 & 0.5849133173 \\ \text { H16 } & -0.2185909906 & 1.1630681851 & 3.7713767860 \\ \text { H17 } & 0.2055635688 & 3.3457122010 & 2.8890567312 \\ \text { C18 } & 0.0005580577 & -2.3173971349 & 0.1493854635 \\ \text { H19 } & 0.9893633675 & -2.0017971020 & 0.4937478751 \\ \text { H20 } & -0.3811619431 & -2.9251016253 & 0.9696038657 \\ \text { C21 } & 0.1127085114 & -3.0998522600 & -1.1522015642 \\ \text { H22 } & -0.8237214585 & -3.6062526660 & -1.4121830282 \\ \text { H23 } & 0.3845891174 & -2.4543298973 & -1.9890663995\end{array}$




$\begin{array}{lrrr}\text { C24 } & -1.7839321477 & -1.4367359289 & 0.1473550670 \\ \text { C25 } & -1.9772260536 & -0.0476385727 & -0.2705211073 \\ \text { H26 } & -2.1179981130 & -2.2181525042 & -0.5307077816 \\ \text { H27 } & -2.2433028874 & 0.1144875010 & -1.3156200893 \\ \text { H28 } & -2.5479584879 & 0.5779468144 & 0.4183214251 \\ \text { H29 } & -2.0330008178 & -1.6335014991 & 1.1862832518 \\ \text { H30 } & 0.8837802958 & -3.8742132318 & -1.0579548551\end{array}$

$\mathrm{N}$

Gas phase Energy: -795.70653671642 hartrees

Solvation Energy: -795.71340068885 hartrees

Zero Point Energy: $150.304 \mathrm{kcal} / \mathrm{mol}$

Coordinates:

$\begin{array}{lccc}\text { Ir1 } & 0.1174408613 & 0.0726326480 & 0.0366811947 \\ \text { O2 } & -0.1550771276 & 0.1572704494 & 2.0622885616 \\ \text { O3 } & 2.3178728745 & 0.1633126707 & 0.4149645808 \\ \text { C4 } & 3.1112075377 & 0.1865036363 & -0.5638032552 \\ \text { C5 } & 2.8103124009 & 0.1252242583 & -1.9421905198 \\ \text { C6 } & 1.5527311520 & 0.0077204755 & -2.5283903131 \\ \text { O7 } & 0.3893562770 & -0.0690145603 & -1.9937997877 \\ \text { H8 } & 1.5258513124 & -0.0373039899 & -3.6243112540 \\ \text { H9 } & 3.6537971201 & 0.1674218721 & -2.6235727653 \\ \text { H10 } & 4.1821762907 & 0.2597092495 & -0.3116903876 \\ \text { O11 } & 0.2425418659 & 2.0499426091 & -0.2219962828 \\ \text { C12 } & 0.1737141414 & 2.8489956675 & 0.7774039679 \\ \text { C13 } & -0.1480851497 & 1.2728641077 & 2.6742581908 \\ \text { C14 } & -0.0056594927 & 2.5559428353 & 2.1280088528 \\ \text { H15 } & 0.2741696074 & 3.9007618727 & 0.4901324503 \\ \text { H16 } & -0.2715706702 & 1.1950465257 & 3.7606461529 \\ \text { H17 } & -0.0287219275 & 3.3937861061 & 2.8157490826 \\ \text { C18 } & -0.8218734059 & -2.3763847789 & 0.0576985125 \\ \text { H19 } & 0.1696891595 & -1.8043886315 & 0.2349686694 \\ \text { H20 } & -0.6794480719 & -3.1394083829 & 0.8336982289 \\ \text { C21 } & -0.7506363243 & -2.9993616842 & -1.3342141534 \\ \text { H22 } & -1.5688615431 & -3.7196119181 & -1.4481149362 \\ \text { H23 } & -0.8450141506 & -2.2394776427 & -2.1126811117 \\ \text { C24 } & -2.1044423860 & -1.5494331036 & 0.3404280272 \\ \text { C25 } & -1.9165379267 & -0.1506333071 & -0.2549481684 \\ \text { H26 } & -2.9763704729 & -2.0983446873 & -0.0451676094 \\ \text { H27 } & -2.1294616871 & -0.1115611194 & -1.3287698272 \\ \text { H28 } & -2.5214634599 & 0.6078283738 & 0.2536693627 \\ \text { H29 } & -2.2274295395 & -1.4660686344 & 1.4248192498 \\ \text { H30 } & 0.1926209033 & -3.5324195488 & -1.4896886411\end{array}$

TS8

Gas phase Energy: - 795.71020013955 hartrees

Solvation Energy: -795.71728484227 hartrees

1 vibrational frequencies below $10.0 \mathrm{~cm}-1$ not included in zero-point

Zero Point Energy: $148.262 \mathrm{kcal} / \mathrm{mol}$

Coordinates:

$\begin{array}{llll}\text { Ir1 } & -0.0274080883 & -0.0390620685 & 0.0045811745 \\ \text { O2 } & -0.1236386265 & -0.0768002114 & 2.0540627792 \\ \text { O3 } & 2.0337640102 & 0.1076047793 & 0.2254333074 \\ \text { C4 } & 2.7885342228 & 0.2291001884 & -0.7911200965\end{array}$




$\begin{array}{lrrr}\text { C5 } & 2.4358739850 & 0.2778203422 & -2.1457349628 \\ \text { C6 } & 1.1424728684 & 0.1755307635 & -2.6656766203 \\ \text { O7 } & 0.0387861893 & 0.0074190445 & -2.0476478091 \\ \text { H8 } & 1.0356292349 & 0.2312903078 & -3.7556570131 \\ \text { H9 } & 3.2425140820 & 0.4034867509 & -2.8596298963 \\ \text { H10 } & 3.8577387826 & 0.3031950680 & -0.5527475921 \\ \text { O11 } & 0.2089491027 & 2.1392923064 & -0.0718562055 \\ \text { C12 } & 0.2491737360 & 2.7922008414 & 1.0073969774 \\ \text { C13 } & -0.0047024800 & 0.9960228384 & 2.7437208963 \\ \text { C14 } & 0.1602091844 & 2.3167411505 & 2.3315997259 \\ \text { H15 } & 0.3676774947 & 3.8814758868 & 0.8950472742 \\ \text { H16 } & -0.0507002796 & 0.8205327819 & 3.8256351254 \\ \text { H17 } & 0.2281555791 & 3.0603415417 & 3.1187195715 \\ \text { C18 } & -0.4757261898 & -2.0773975623 & 0.0655456632 \\ \text { H19 } & -0.2756335191 & -2.4541941231 & 1.0710472080 \\ \text { C20 } & -1.7772886047 & -1.4052058326 & -0.0708288127 \\ \text { H21 } & -1.6961841458 & -0.0161914371 & -0.0946361303 \\ \text { H22 } & -2.2865639126 & -1.4962048259 & -1.0307384639 \\ \text { H23 } & -2.4491270992 & -1.4193132467 & 0.7870680386 \\ \text { C24 } & 0.0252831874 & -2.9964737725 & -1.0339465652 \\ \text { H25 } & 1.1204305983 & -3.0458942413 & -0.9793683521 \\ \text { H26 } & -0.2181199013 & -2.5650038707 & -2.0113529487 \\ \text { C27 } & -0.5503246799 & -4.4188968532 & -0.9334643076 \\ \text { H28 } & -0.1546390371 & -5.0630155103 & -1.7263621078 \\ \text { H29 } & -1.6429954680 & -4.4137548557 & -1.0216110780 \\ \text { H30 } & -0.2981948934 & -4.8800127385 & 0.0282642025\end{array}$

O

Gas phase Energy: - 795.73024259843 hartrees

Solvation Energy: -795.73725516927 hartrees

Zero Point Energy: $149.616 \mathrm{kcal} / \mathrm{mol}$

Coordinates:

$\begin{array}{lrrr}\text { Ir1 } & -0.0145559963 & -0.0443457500 & 0.0171491736 \\ \text { O2 } & -0.1134437516 & -0.0582400639 & 2.0668123372 \\ \text { O3 } & 2.1802130536 & 0.1347982288 & 0.2198753265 \\ \text { C4 } & 2.8723695684 & 0.4005025094 & -0.8038612852 \\ \text { C5 } & 2.4618933286 & 0.4901572645 & -2.1470999915 \\ \text { C6 } & 1.1768679272 & 0.2757561070 & -2.6481542616 \\ \text { O7 } & 0.0903442051 & -0.0162274281 & -2.0401940931 \\ \text { H8 } & 1.0567479333 & 0.3493815893 & -3.7364160288 \\ \text { H9 } & 3.2294290553 & 0.7292915979 & -2.8755555531 \\ \text { H10 } & 3.9445024154 & 0.5719619313 & -0.6158594542 \\ \text { O11 } & 0.1410731525 & 2.0300445072 & -0.1158761490 \\ \text { C12 } & 0.1502031184 & 2.7432375774 & 0.9386205352 \\ \text { C13 } & -0.0457622347 & 1.0239144743 & 2.7369143465 \\ \text { C14 } & 0.0663561102 & 2.3396363717 & 2.2761549273 \\ \text { H15 } & 0.2388252547 & 3.8211875088 & 0.7507403256 \\ \text { H16 } & -0.0865499846 & 0.8756608834 & 3.8226621180 \\ \text { H17 } & 0.1011556186 & 3.1205951888 & 3.0276329676 \\ \text { C18 } & 0.3906444866 & -2.2062281694 & 0.1773014873 \\ \text { H19 } & 0.7988145051 & -2.3141535022 & 1.1817074342 \\ \text { C20 } & -0.9934134160 & -2.0017286102 & 0.0658488696 \\ \text { H21 } & -1.4940439358 & -2.1838721986 & -0.8815466089 \\ \text { H22 } & -1.6265829563 & -2.0182428876 & 0.9469542593\end{array}$




$\begin{array}{lrrr}\text { C23 } & 1.2230383734 & -2.8210193987 & -0.9251040086 \\ \mathrm{H} 24 & 2.2243236561 & -2.3786068686 & -0.9260267089 \\ \mathrm{H} 25 & 0.7698266617 & -2.5975874928 & -1.8963050341 \\ \mathrm{C} 26 & 1.3436260625 & -4.3446131330 & -0.7445241841 \\ \mathrm{H} 27 & 1.9618820093 & -4.7805907013 & -1.5362086434 \\ \mathrm{H} 28 & 0.3609062009 & -4.8270049639 & -0.7778011930 \\ \mathrm{H} 29 & 1.8057156077 & -4.5958662045 & 0.2167460168 \\ \mathrm{H} 30 & -1.5651850516 & 0.1689777308 & -0.0882052466\end{array}$

